\title{
NLTE AND LTE LICK INDICES FOR RED GIANTS FROM [Fe/H] 0.0 TO -6.0 AT SDSS AND IDS SPECTRAL RESOLUTION
}

\author{
C. Ian Short, Mitchell E. Young, and Nicholas Layden \\ Department of Astronomy \& Physics and Institute for Computational Astrophysics, Saint Mary’s University, Halifax, NS, B3H 3C3, Canada; ishort@ap.smu.ca \\ Received 2015 May 14; accepted 2015 July 29; published 2015 September 1
}

\begin{abstract}
We investigate the dependence of the complete system of 22 Lick indices on overall metallicity scaled from solar abundances, $[\mathrm{M} / \mathrm{H}]$, from the solar value, 0.0 , down to the extremely metal-poor (XMP) value of -6.0 , for latetype giant stars (MK luminosity class III, $\log g=2.0)$ of MK spectral class late-K to late-F $\left(3750<T_{\text {eff }}<6500\right.$ $\mathrm{K})$ of the type that are detected as "fossils" of early galaxy formation in the Galactic halo and in extra-galactic structures. Our investigation is based on synthetic index values, $I$, derived from atmospheric models and synthetic spectra computed with PHOENIX in Local Thermodynamic Equilibrium (LTE) and Non-LTE (NLTE), where the synthetic spectra have been convolved to the spectral resolution, $R$, of both IDS and SDSS (and LAMOST) spectroscopy. We identify nine indices, that we designate "Lick-XMP," that remain both detectable and significantly $[\mathrm{M} / \mathrm{H}]$-dependent down to $[\mathrm{M} / \mathrm{H}]$ values of at least $\sim-5.0$, and down to $[\mathrm{M} / \mathrm{H}] \sim-6.0$ in five cases, while also remaining well-behaved (single-valued as a function of $[\mathrm{M} / \mathrm{H}]$ and positive in linear units). For these nine indices, we study the dependence of $I$ on NLTE effects, and on spectral resolution. For our LTE $I$ values for spectra of SDSS resolution, we present the fitted polynomial coefficients, $C_{\mathrm{n}}$, from multi-variate linear regression for $I$ with terms up to third order in the independent variable pairs $\left(T_{\text {eff }},[\mathrm{M} / \mathrm{H}]\right)$ and $(V-K,[\mathrm{M} / \mathrm{H}])$, and compare them to the fitted $C_{\mathrm{n}}$ values of Worthey et al. at IDS spectral resolution. For this fitted $I$ data-set we present tables of LTE partial derivatives, $\left.\frac{\partial I}{\partial T_{\text {eff }}}\right|_{[\mathrm{M} / \mathrm{H}]},\left.\frac{\partial I}{\partial[\mathrm{M} / \mathrm{H}]}\right|_{T_{\text {eff }}},\left.\frac{\partial I}{\partial(V-K)}\right|_{[\mathrm{M} / \mathrm{H}]}$, and $\left.\frac{\partial I}{\partial[\mathrm{M} / \mathrm{H}]}\right|_{(V-K)}$, that can be used to infer the relation between a given difference, $\Delta I$, and a difference $\Delta T_{\text {eff }}$ or $\Delta(V-K)$, or a difference $\Delta[\mathrm{M} / \mathrm{H}]$, while the other parameters are held fixed. For Fe-dominated Lick indices, the effect of NLTE is to generally weaken the value of $I$ at any given $T_{\text {eff }}$ and $[\mathrm{M} / \mathrm{H}]$ values. As an example of the impact on stellar parameter estimation, for latetype giants of inferred $T_{\text {eff }} \gtrsim 4200 \mathrm{~K}$, an Fe-dominated $I$ value computed in LTE that is too strong might be compensated for by inferring a $T_{\text {eff }}$ value that is too large.
\end{abstract}

Key words: stars: atmospheres - stars: fundamental parameters - stars: late-type

Supporting material: machine-readable tables

\section{INTRODUCTION}

The determination of stellar parameters, especially overall metallicity (denoted here $[\mathrm{M} / \mathrm{H}]$ unless otherwise indicated) and detailed abundances of individual metals, in stars of remote Galactic and extra-galactic structures has become crucial to the study of galaxy formation and evolution, including that of the Milky Way. Metallicity distribution functions for galaxies and globular star clusters (GCs) reveal information about multiple populations from multiple star formation episodes and allow the investigation of the history of star formation. Chemical tagging of stellar populations allows the investigation of the link between Galactic structures such as GCs and nearby extragalactic ones such as ultra-faint dwarf and dwarf spheroidal satellite galaxies, and the process through which galaxies are assembled in hierarchical structure formation (see Mucciarelli et al. 2013 for a recent example, and Frebel \& Norris 2013 and Belokurov 2013 for reviews). However, stars in remote structures are often only significantly and efficiently detectable with low- to moderate-resolution spectroscopy, such as that of the Sloan Digital Sky Survey-Sloan Extension for Galactic Understanding and Exploration (SDSS-SEGUE), that precludes the measurement of individual spectral lines, and usefully accurate $[\mathrm{M} / \mathrm{H}]$ values must be obtained from observationally expensive follow-up spectroscopy at high spectral resolution (e.g., see Aoki et al. 2013). Ivezic et al. (2012) contains a recent authoritative review of how modest resolution spectroscopic surveys are revolutionizing our study of Galactic populations and leading to insights into Galactic formation. As a result, there is active interest in novel methods for extracting $[\mathrm{M} / \mathrm{H}]$ and $T_{\text {eff }}$ values from low- to moderateresolution data (see Schlaufman \& Casey 2014 for a recent investigation of a method based on IR molecular bands, and Miller 2015 for a very recent investigation of a photometric method based on SDSS ugriz photometry.)

The original system of 11 (Gorgas et al. 1993), and then 21 (Worthey et al. 1994, W94 henceforth), Lick/IDS spectral indices, $I$, was defined to optimize the determination of $[\mathrm{Fe} / \mathrm{H}]$ and, hence, age $(t)$, from integrated light (IL) spectra of faint spatially unresolved old stellar populations $(-1<[\mathrm{M} / \mathrm{H}]<$ $+0.5,0.5<t<20 \mathrm{Gyr})$ dominated by $\mathrm{G}$ and $\mathrm{K}$ stars, obtained at low spectral resolution $(R<1000)$ in the $\lambda 4000$ to $6200 \AA$ spectral band. (Worthey \& Ottaviani 1997 explored four new indices based on two definitions, each of $\mathrm{H} \gamma$ - and $\mathrm{H} \delta$ centered features, but found them to be of limited usefulnessa result that is consistent with our own investigation.) Because of the emphasis on old stellar populations, such as that of GCs and "early-type" galaxies, red giant (RGB) stars are important contributors to the IL spectrum. The indices were discovered by empirically identifying composite spectral features in low $R$ spectra of Galactic and GC G and $\mathrm{K}$ stars that showed a significant and useful correlation with one of $[\mathrm{M} / \mathrm{H}], T_{\text {eff }}$, or $\log g$ while (hopefully) depending less sensitively on the other two. The original Lick/IDS system was defined with spectra obtained with the Lick Observatory Image Dissector Scanner 
(IDS) having a spectral sampling, $\Delta \lambda$, of $\sim 8 \AA$ in a region centered at $5200 \AA$ spanning a $\Delta \lambda$ range of $2400 \AA$, corresponding to a spectral resolution $R \equiv \lambda / \Delta \lambda$ of $\sim 650$.

Given the usefulness of the Lick indices for modern moderate resolution spectroscopic surveys such as SDSSSEGUE and LAMOST, Franchini et al. (2010) developed the system further by creating a synthetic library of $I$ values for dwarf and giant stars derived from synthetic spectra that had been convolved to the higher SDSS $R$ value of 1800, and tested the predicted relationship between the $I$ values and stellar parameters against that of several empirical spectral libraries, including the SDSS-DR7 spectroscopic database itself. They also supplemented the 21 indices of W94 with a new near-UV index, namely $\mathrm{CaHK}$ - a prominent spectral feature that has proved useful for identifying candidate extremely metal-poor (XMP) stars in productive surveys such as the HK survey of Beers et al. (1992). One of the main conclusions of Franchini et al. (2010) is that $T_{\text {eff }}$ values derived from fitting their synthetic indices to SDSS-SEGUE spectra of late-type giants were systematically lower than the $T_{\text {eff }}$ values derived with the SEGUE Stellar Parameter Pipeline.

Many of the Lick indices are dominated by, or have significant contributions from, lines of $\mathrm{Fe}$ I. Initial investigations of metal-poor stars in which the Fe I extinction is treated with Non-local Thermodynamic Equilibrium (Non-LTE, NLTE) indicate that NLTE effects become increasingly important with decreasing metallicity, and alter the inferred $T_{\text {eff }}$ values by as much as $400 \mathrm{~K}$ and the derived abundances of Fe by 0.4 dex for metal-poor giants with parameters based on RAVE survey spectra (Ruchti et al. 2013; Serenelli et al. 2013). Recently, Short \& Campbell (2013) and papers in that series have found that when most of the light and Fe-group metals that contribute to the visible band spectral line blanketing of mildly metal-poor RGB stars is treated in NLTE, the $T_{\text {eff }}$ value inferred from spectrophotometric spectral energy distributions (SEDs) of $R \approx 100$ is reduced by $\gtrsim 50 \mathrm{~K}$. Therefore, calibration of the Lick $I([\mathrm{M} / \mathrm{H}])$ relation based on NLTE modeling may be crucial to using the indices for accurate $[\mathrm{M} / \mathrm{H}]$ inference.

Our goal is to extend an analysis of the detectability and sensitivity to stellar parameters, including $[\mathrm{M} / \mathrm{H}]$, of the Lick indices to the regime of XMP red giants, and to investigate the magnitude of NLTE effects on the value of modeled Lick indices. In Section 2, we describe the atmospheric models and the spectrum synthesis, and the procedure for producing synthetic $I$ values. In Section 3, we identify the Lick indices that remain most useful at XMP metallicities, and provide useful polynomial fits and partial derivatives for index values, $I$, modeled in LTE, in terms of $T_{\text {eff }}, V-K$, and $[\mathrm{M} / \mathrm{H}]$. In Section 4, we present conclusions.

\section{MODELING}

\subsection{Model Grid}

We have used PHOENIX V. 15 (Hauschildt et al. 1999) to compute a grid of atmospheric models and corresponding synthetic spectra in both LTE and "massive multi-species NLTE" (Short \& Hauschildt 2009) for very-metal-poor (VMP) and XMP red (and "orange") giant stars of MK spectral class late-F to late- $\mathrm{K}$, covering the range of $T_{\text {eff }}$ and $[\mathrm{M} / \mathrm{H}]$ values of stars that are spectroscopically accessible at Galactic halo distances and that serve as useful stellar "fossils" for Galactic

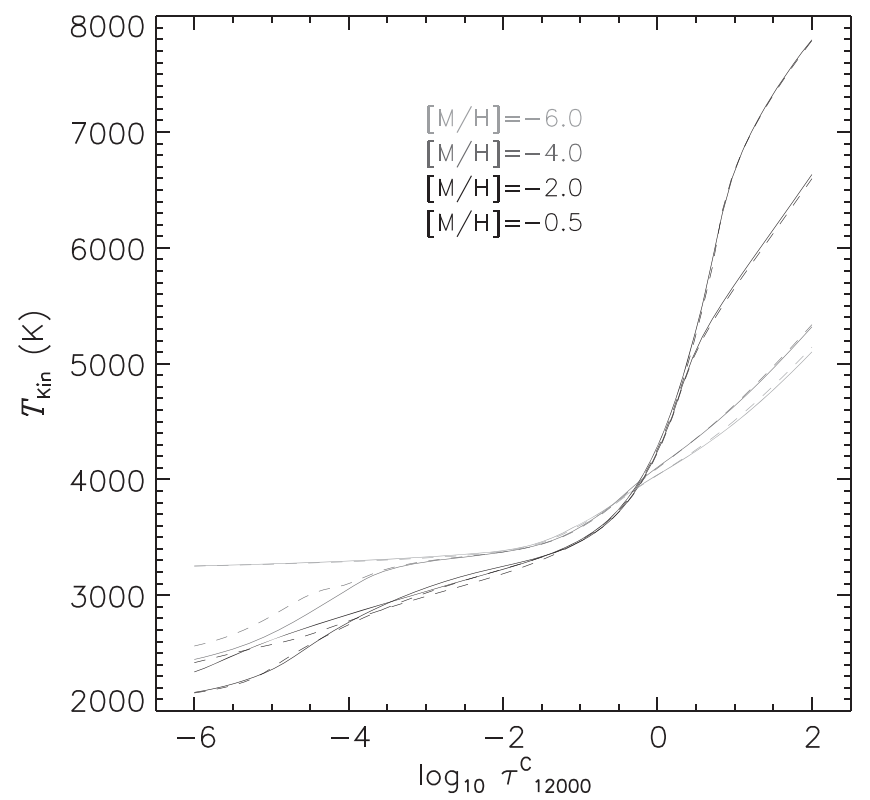

Figure 1. $T_{\mathrm{Kin}}\left(\tau_{12000}\right)$ structures for models of $T_{\text {eff }}=4000 \mathrm{~K}$ and $[\mathrm{M} / \mathrm{H}]$ values of $-0.5,-2.0,-4.0$, and -6.0 . Gray-scale: darker lines indicates larger $[\mathrm{M} / \mathrm{H}]$ values. Results are shown for LTE (dashed line) and NLTE (solid line) models.

archeology (Cohen et al. 2013). The grid also includes red giants of a higher $[\mathrm{M} / \mathrm{H}]$ value representative of the solar neighborhood and disk population for comparison. The parameters of the LTE grid are $3750 \leqslant T_{\text {eff }} \leqslant 6500 \mathrm{~K}$ with $\Delta T_{\text {eff }}=250 \mathrm{~K},-6.0 \leqslant[\mathrm{M} / \mathrm{H}] \leqslant 0.0$ with $\Delta[\mathrm{M} / \mathrm{H}]=1.0$ for $[\mathrm{M} / \mathrm{H}]<-2.0$ and 0.5 for $[\mathrm{M} / \mathrm{H}] \geqslant-2.0, \log g=2.0$

Figure 1 shows the $T_{\text {Kin }}\left(\tau_{12000}\right)$ structure of a subset of our models for $T_{\text {eff }}=4000 \mathrm{~K}$ and $[\mathrm{M} / \mathrm{H}]$ values of $-0.5,-2.0$, -4.0 , and -6.0 , where $\tau_{12000}$ is the monochromatic continuum optical depth at $12000 \AA$ and serves as our standard radial depth variable. The reduction in the well-understood backwarming and surface cooling effects caused by line extinction as $[\mathrm{M} / \mathrm{H}]$ decreases is readily noticeable. For comparison, the grid of Franchini et al. (2010) has $3500 \leqslant T_{\text {eff }} \leqslant 7000 \mathrm{~K}$ with $\Delta T_{\text {eff }}=250 \mathrm{~K},-2.5 \leqslant[\mathrm{Fe} / \mathrm{H}] \leqslant 0.5$ with $\Delta[\mathrm{Fe} / \mathrm{H}]=0.5$ generally, with the addition of $[\mathrm{Fe} / \mathrm{H}]=-4.0$ for their $\alpha$ enhanced models, and $0.5 \leqslant \log g \leqslant 5.0$ with $\Delta \log g=0.5$, where $[\mathrm{Fe} / \mathrm{H}]$ denotes the scaled abundance parameter for elements other than $\alpha$-process elements. The most important distinguishing features of our grid are the extension to $[\mathrm{M} / \mathrm{H}]$ values of -6.0 , and the inclusion of NLTE models for a subset of parameter values spanning the grid. Although there are few stars of $[\mathrm{M} / \mathrm{H}] \lesssim-3.5$, even among halo stars useful for Galactic archeology, extending the grid to $[\mathrm{M} / \mathrm{H}]=-6.0$ allows us to anchor the $I([\mathrm{M} / \mathrm{H}])$ fit through the useful $[\mathrm{M} / \mathrm{H}]$ range.

Because of the large number of $[\mathrm{M} / \mathrm{H}]$ values (nine) and doubling of most of the grid to include NLTE counterparts, the number of models was limited by fixing the $\log g$ value at 2.0, representative of the giant population, and giving all models a scaled-solar abundance distribution based on the abundances of Grevesse \& Sauval (1998; therefore, for our grid the overall metallicity parameter, $[\mathrm{M} / \mathrm{H}]$, is identical with the $[\mathrm{Fe} / \mathrm{H}]$ parameter). W94 and Gorgas et al. (1993) found that important $T_{\text {eff }}-$ and $[\mathrm{Fe} / \mathrm{H}]$-sensitive $I$ values were least sensitive to $\log g$. Nevertheless, the lack of the $\log g$ dimension in our $I$ polynomial fits described below leads to polynomial fitting 
Table 1

LTE Index Values, $I$, at SDSS Resolution for the Lick-XMP Indices $T_{\text {eff }}=400 \mathrm{~K}$ and Select $[\mathrm{Fe} / \mathrm{H}]$ Values with Scaled Solar and with $\alpha$-enhanced Abundances (see text)

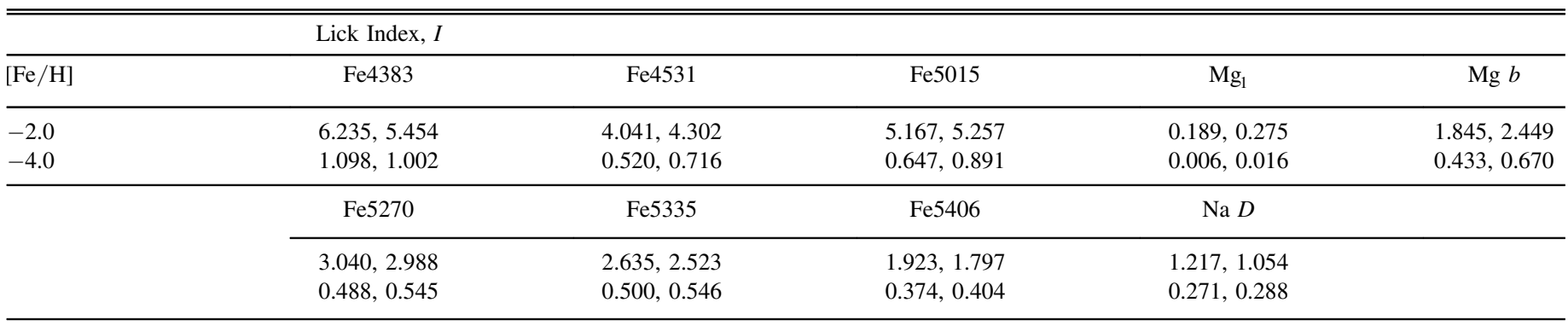

Note. We note that for the scaled-solar abundance models, $[\mathrm{Fe} / \mathrm{H}]$ is identical to our $[\mathrm{M} / \mathrm{H}]$ grid parameter. The first and second numbers in each comma-separated pair refer to scaled solar and $\alpha$-enhanced abundances.

coefficients that are not directly comparable to those of W94 from their analysis of observed IDS spectra. Adopting an $\alpha$-enhancement of 0.0 is expected to have a minor effect on the differential comparison of most Fe-dominated $[\mathrm{M} / \mathrm{H}]$-sensitive $I$ values computed in LTE and NLTE (because these are really more $[\mathrm{Fe} / \mathrm{H}]$ indicators, and $\mathrm{Fe}$ is not an $\alpha$-element), and will allow direct assessment of pure NLTE effects on $I$ values across the entire range of the $[\mathrm{M} / \mathrm{H}]$ value. Moreover, incorporating the $\alpha$-enhancement requires a model for how the value of the enhancement increases with a decreasing $[\mathrm{M} / \mathrm{H}]$ value in the range of 0.0 to -1.0 . Nevertheless, a future direction is to extend our grid in the $\alpha$-enhancement dimension. As a preliminary assessment of the effect of $\alpha$-enhancement, in Table 1 we present the computed values of the index, I, computed at SDSS spectral resolution for our subset of nine "Lick-XMP" indices (see below) for LTE models of $T_{\text {eff }}=4000 \mathrm{~K}, \log g=2.0$, for which the $I$ values are relatively strong, and select $[\mathrm{Fe} / \mathrm{H}]$ values of -2.0 and -4.0 and scaled solar abundance, and abundances with the maximal relative enhancement of +0.4 of the eight $\alpha$-process elements of even atomic numbers from $\mathrm{O}(Z=8)$ to $\mathrm{Ti}(Z=22)$. $\mathrm{Mg}$ is an $\alpha$-process element, and the $\mathrm{Mg}_{1}$ and $\mathrm{Mg} b$ indices are more strongly affected, increasing by a factor of $\sim 1.5$ at $[\mathrm{Fe} / \mathrm{H}]=-2$ where their value is still large. Of our nine Lick-XMP indices, the results presented below for $\mathrm{Mg}_{1}$ and $\mathrm{Mg}$ $b$ should be regarded as most suspect and require a follow up investigation with full $\alpha$-enhanced NLTE and LTE model grids. The inclusion of NLTE effects in the modeling is not likely to change the conclusion that non- $\alpha$-element spectral features are significantly less affected by $\alpha$-enhancement than $\alpha$-element spectral features are. As might be expected, the effect of $\alpha$ enhancement on the Fe-dominated indices Fe4531, Fe5015, Fe5270, Fe5335, and the $\mathrm{Na} D$ index is minor, being of the order of $10 \%$ or less. We note that for some non- $\alpha$ element indices, the effect of $\alpha$-enhancement can be to reduce the index slightly at some $[\mathrm{Fe} / \mathrm{H}]$ values, while increasing it at others. Changes to the composition can affect the spectrum in the bracketing pseudo-continuum windows that define any index, and will also indirectly affect the strength of features contributing to any index through the effect on the electron number density.

Our models have spherical geometry with radii based on an adopted mass of $M=1 M_{\odot}$, a microturbulence broadening parameter of $\xi_{\mathrm{T}}=2.0 \mathrm{~km} \mathrm{~s}^{-1}$, which is consistent with what has been measured and adopted for late-type giants generally, and a mixing-length parameter for the treatment of convective energy transport, $l$, of $1.0 H_{\mathrm{P}}$ (pressure scale heights).

\subsubsection{NLTE treatment}

We treat 6706 atomic energy-levels (E-levels) connected by 74550 bound-bound $(b-b)$ transitions of 35 chemical species accounting for various ionization stages of 20 chemical elements, including $\mathrm{H}, \mathrm{He}, \mathrm{CNO}$, and the Fe-group elements that blanket late-type visible band stellar spectra, as well as other abundant light metals. We compute the NLTE level populations, $n_{\mathrm{i}}\left(\tau_{12000}\right)$, and hence the corresponding extinction coefficient, $\kappa_{\lambda}\left(\tau_{12000}\right)$, in self-consistent multi-level NLTE by solving the system of coupled rate equations of statistical equilibrium consistently with the equation of radiative transfer (RT) in each of the relevant bound-bound $(b-b)$ and boundfree $(b-f)$ transitions. Short \& Hauschildt (2009) contains a description of the species treated in NLTE, sources of atomic data, and other important details.

Because NLTE models are more computationally expensive, we only produced NLTE models and spectra at a subset of our LTE grid, as follows $4000 \leqslant T_{\text {eff }} \leqslant 6500 \mathrm{~K}$ with $\Delta T_{\text {eff }}=500$ $\mathrm{K}, \quad-6.0 \leqslant[\mathrm{M} / \mathrm{H}] \leqslant 0.0$ with $\Delta[\mathrm{M} / \mathrm{H}]=1.0$, with the addition of $[\mathrm{M} / \mathrm{H}]=-0.5$.

This is sufficient to assess the dependence of NLTE effects on Lick indices throughout the grid. Figure 1 shows NLTE $T_{\text {Kin }}\left(\tau_{12000}\right)$ structures for comparison with those of LTE. NLTE radiative equilibrium is complex, though, Anderson (1989) contains a very thorough analysis for the case of the Sun, and Short et al. (2012) extends the analysis to solar metallicity and moderately metal-poor RGB stars.

NLTE Fe treatment: the predicted magnitude of the wellknown Fe I NLTE "over-ionization," and the resulting predicted brightening of the Fe I-blanketed near-UV and blue spectral bands with respect to the rest of the SED (see Rutten 1986; Short \& Hauschildt 2009) depends on the details of the atomic model of Fe I used in the NLTE Fe treatment. More specifically, the completeness with which high-energy $E$-levels are included near the ionization limit, $\chi_{\mathrm{I}}$, affects the computed rate of collisional recombination from $\mathrm{Fe}$ II, and thus the $\mathrm{Fe}_{\mathrm{I}}$ / Fe II ionization equilibrium (Mashonkina et al. 2011). Generally, the more $E$-levels are included for which the atomic energy gap, $\Delta \chi$, between the $E$-level and $\chi_{\mathrm{I}}$ is less than the average collisional energy among particles $(k T)$ throughout the line-forming region of the atmosphere, the more accurate the NLTE effect on the computed SED will be. For Fe I, 
$\chi_{\mathrm{I}}=7.9024 \mathrm{eV}$, and in our 494-level model $\mathrm{Fe}_{\mathrm{I}}$ atom, the highest lying $E$-level has $\chi=7.538 \mathrm{eV}$, for a minimum $\Delta \chi$ gap of $0.364 \mathrm{eV}$. The line-forming region of the atmosphere throughout the visible band generally lies at shallower total optical depths, $\tau_{\lambda}$, than the layer where the continuum value of $\tau_{\lambda}$ is unity, where $T\left(\tau_{\lambda}\right) \leqslant T_{\text {eff }}$. For the warmest models in our grid $\left(T_{\text {eff }}=6500 \mathrm{~K}\right), k T \leqslant 0.560 \mathrm{eV}$ in the line forming region, and we have eight $E$-levels for which $\Delta \chi<k T$, at least in the lower line-forming region. For the coolest models in our grid $\left(T_{\text {eff }}=3750 \mathrm{~K}\right), k T \leqslant 0.323 \mathrm{eV}$ in the line forming region, and we just miss having any E-levels for which $\Delta \chi<k T$. We expect our prediction of NLTE Fe I effects to be most accurate at the warm end of our grid where collisional recombination into our highest $E$-levels is energetically accessible. At the cool end, the collisional recombination rate is artificially suppressed by the lack of higher-lying $E$-levels in the model atom. The recombination rate is underestimated for our cooler models, and the NLTE over-ionization effect is likely overestimated. Our NLTE modeled NLTE effects on I values may be thought of, cautiously, as upper limits. We plan to expand the PHOENIX NLTE Fe I atom in the near future, but this is a significant project in its own right.

\subsection{Synthetic Spectra}

Our longer-term goal is to identify useful $T_{\text {eff }}$ and overall $[\mathrm{M} / \mathrm{H}]$ line diagnostics for high spectral resolution from the near-UV to the the near-IR (NIR). Therefore, we have computed synthetic spectra for each of our models for $3000<\lambda<26000 \AA$ with a spectral sampling, $\Delta \lambda$, set so as to maintain an $R$ value of 300,000 throughout, sufficient to fully resolve spectral line cores. This $\lambda$ range includes the NIR $J, H$, and $K$ photometric bands, in which useful line lists of stellar parameter and abundance diagnostics have recently been published (Le et al. 2011; Bergemann et al. 2012; Cesetti et al. 2013).

Our synthetic spectra were post-processed by broadening with a Gaussian kernel to $R$ values of 650 and, following Franchini et al. (2010), 1800 to match the resolution of the original IDS, and SDSS-SEGUE spectroscopy, respectively. A Gaussian is only an approximation to the real instrumental spectral profiles of IDS and SDSS-SEGUE spectroscopy, but at this stage our study is a differential one to compare the effect of NLTE on Lick indices to that of the choice of $R$ value as a function of $[\mathrm{M} / \mathrm{H}]$. We do not account for either macroturbulent or rotational broadening. Rotation is expected to be modest in evolved stars of large radius, and both effects are expected to be minor at these $R$ values. Figure 2 shows representative synthetic spectra for the models of Figure 1 convolved to IDS spectral resolution, with the Lick indices labeled, and it can be seen that some of the strongest spectral features are still significant at $[\mathrm{M} / \mathrm{H}]$ values as low as -6.0 . Figure 3 shows the relative flux difference at IDS resolution, $\Delta F_{\lambda} \equiv\left(F_{\lambda, \mathrm{NLTE}}-F_{\lambda, \mathrm{LTE}}\right) / F_{\lambda_{\mathrm{LTE}}}$, for models of $T_{\text {eff }}=4000 \mathrm{~K}$, and $[\mathrm{M} / \mathrm{H}]$ values of $0.0,-2.0$, and -6.0 , with the Lick indices labeled. $\Delta F_{\lambda}$ is generally positive at the $\lambda$ values of the Lick indices because most low- $\chi$ spectral lines from neutral ionization stages of metals are weaker in NLTE than in LTE.

\subsection{Synthetic Lick Indices}

We use our LTE and NLTE synthetic spectra, convolved to both IDS and SDSS spectral resolution, to compute LTE and

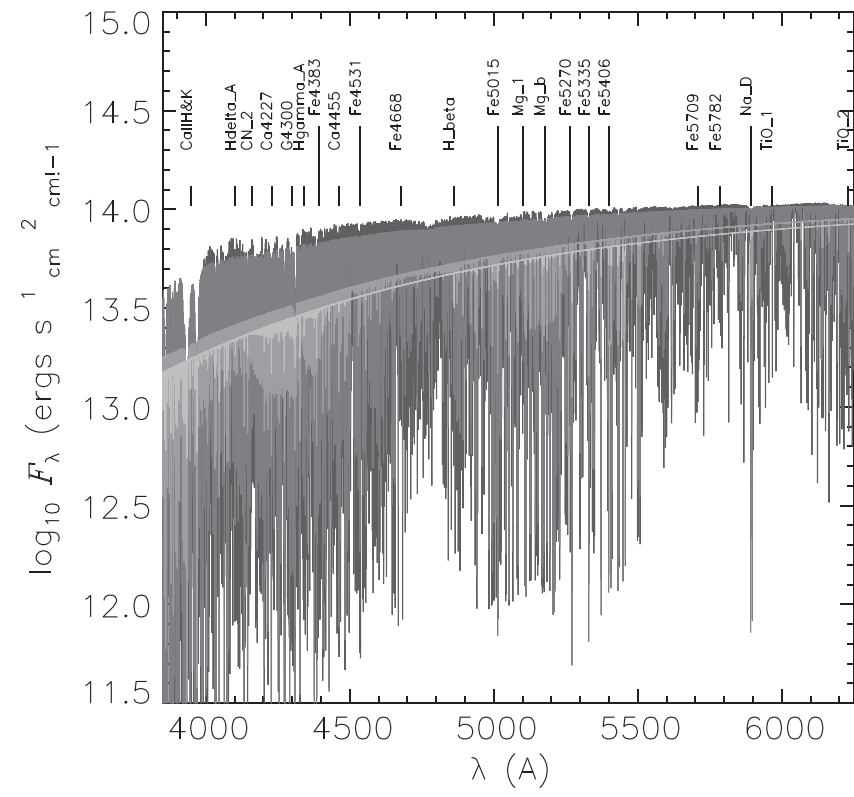

Figure 2. Synthetic spectra for the LTE models of Figure 1, convolved to IDS spectral resolution for clarity. The grayscale is the same as in Figure 1. Index labels that are offset upward refer to our nine Lick-XMP indices.

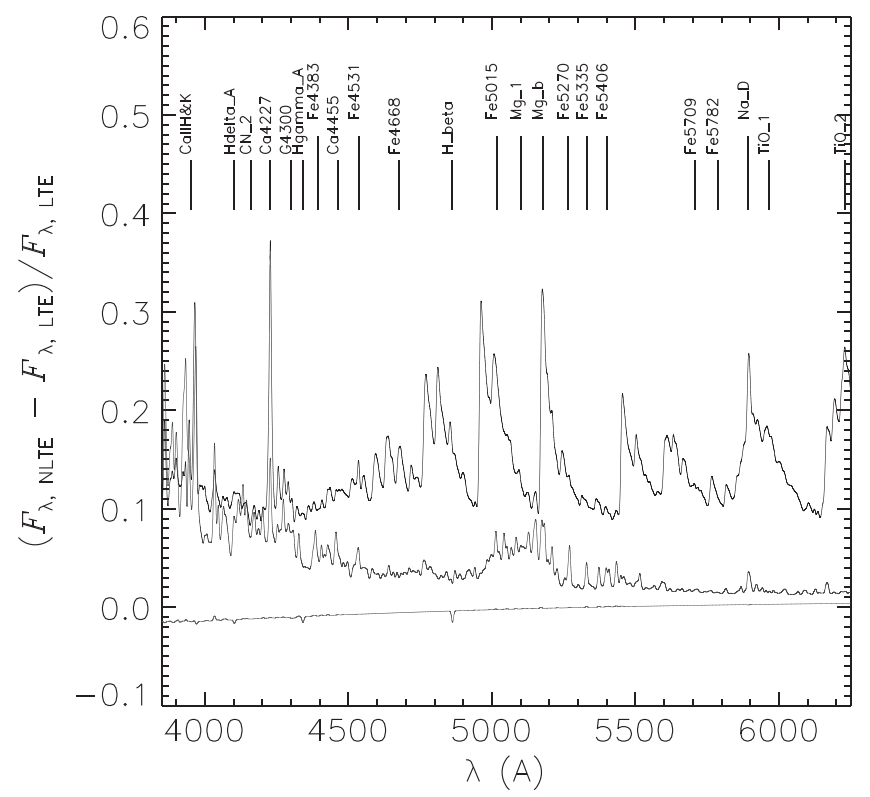

Figure 3. Relative difference of synthetic spectra convolved to IDS resolution showing the comparison of NLTE to LTE results for models of $T_{\text {eff }}=4000 \mathrm{~K}$ and $[\mathrm{M} / \mathrm{H}]$ values of $-0.5,-2.0$, and -6.0 . The grayscale and index label positions are the same as in Figure 2.

NLTE IDS and SDSS Lick indices, $I$, following the prescription of W94. We took the $\lambda$ values defining the latest recommended index and associated pseudo-continuum bands, similar to the information presented in Table 1 of W94, from the official Lick index Web site (http://astro.wsu.edu/ worthey $/ \mathrm{html} /$ system.html), as did Franchini et al. (2010). The IDS indices conform to the well-studied Lick index system as originally defined and are directly comparable to those of Gorgas et al. (1993) and W94. They serve as a check on our procedures as well as allowing us to assess the impact of NLTE effects at IDS resolution. The SDSS indices are comparable to those of Franchini et al. (2010) and allow for an assessment of 
NLTE effects at a somewhat higher resolution typical of more modern spectroscopic surveys such as SDSS and LAMOST. Furthermore, comparing the LTE IDS and SDSS indices allows for an assessment of the dependence of the $I$ sensitivity to $T_{\text {eff }}$ and $[\mathrm{M} / \mathrm{H}]$ on the $R$ value.

Following Gorgas et al. (1993) and W94, we also computed the photometric $V-K$ index for the models as an observational surrogate for the independent parameter $T_{\text {eff }} . V-K$ has been found to be relatively insensitive to $\log g$ and $[\mathrm{M} / \mathrm{H}]$, and a good proxy for $T_{\text {eff }}$ over the GK star range (Bell \& Gustafsson 1989). For consistency with W94, we use the $V$ and $K$-band filter definitions of Johnson et al. (1966) and calibrate the index with a single-point calibration of the $[\mathrm{Fe} / \mathrm{H}]=0.0$ models at $T_{\text {eff }}=4000 \mathrm{~K}$ to the $(V-K)-T_{\text {eff }}$ relation given in Table 4 of Ridgway et al. (1980). The Ridgway et al. (1980) $(V-K)-T_{\text {eff }}$ relation is for giant stars, and a $T_{\text {eff }}$ value of $4000 \mathrm{~K}$ is near the center of their calibrated $T_{\text {eff }}$ range, and overlaps with the $T_{\text {eff }}$ range of our grid. We always use the LTE model $V-K$ color on the grounds that it is serving as an independent variable in this analysis, and the LTE grid is more complete.

It is worth reiterating remarks made by previous investigators about the particular diagnostic utility of those indices that are expected to be most useful in this investigation:

Fe4383 and Fe4668 (W94) and Fe5270 and Fe5335 (Gorgas et al. 1993) are $[\mathrm{Fe} / \mathrm{H}]$-sensitive with a range in $I$ value significantly greater than measurement uncertainty, and are expected to be especially useful here if they remain detectable down to XMP metallicities (note that Fe4668 has significant contributions from $\mathrm{Mg}, \mathrm{Cr}, \mathrm{Ti}$, and $\mathrm{C}_{2}$ ). $\mathrm{Ca} 4227$ is dominated by $\mathrm{Ca}$ (whereas $\mathrm{Ca} 4455$ is more influenced by Fe-group lines), thus providing one of the few atomic indices not heavily affected by $\mathrm{Fe}$, and is somewhat sensitive to overall $[\mathrm{M} / \mathrm{H}]$ (W94) as well as $[\alpha / \mathrm{H}]$ given that $\mathrm{Ca}$ is an $\alpha$-process element. $\mathrm{CN}_{2}$ is a modification of $\mathrm{CN}_{1}$ designed to avoid contamination from the $\mathrm{H} \delta$ line and is strongly dependent on overall $[\mathrm{M} / \mathrm{H}]$ for giants (but less so for dwarfs; W94). By contrast to the preceding, $\mathrm{H} \beta$ has been found to be most strongly dependent on $T_{\text {eff }}$ (Gorgas et al. 1993), and thus provides a valuable complementary diagnostic. Indices $\mathrm{Mg}_{1}$ (dominated by $\mathrm{MgH}$ ) and $\mathrm{Mg} b$ were found by Gorgas et al. (1993) to be usefully sensitive to $\log g$, and we include them in our investigation to re-assess their $T_{\text {eff }}$ and $[\mathrm{M} / \mathrm{H}]$ sensitivity (note that $\mathrm{Mg}_{2}$ includes contributions from both $\mathrm{MgH}$ and the $\mathrm{Mg} b$ lines, and thus is a less pure signal of both). $\mathrm{Na} D$ is known to be significantly contaminated by interstellar (ISM) extinction, which complicates its interpretation (Gorgas et al. 1993).

Franchini et al. (2010) investigated the influence of enhanced $\alpha$-element abundances on model $I$ values. They found that for $T_{\text {eff }}>4250 \mathrm{~K}$ the most $\alpha$-sensitive indices are $\mathrm{CN}_{1}, \mathrm{CN}_{2}$, $\mathrm{CaHK}, \mathrm{Ca} 4227, \mathrm{Fe} 4668, \mathrm{Mg}_{1}, \mathrm{Mg}_{2}$, and $\mathrm{Mg} b$, and that the least $\alpha$-sensitive indices are G4300, Ca4455, Fe4531, Fe5015, Fe5782, and $\mathrm{H} \beta$. For $T_{\text {eff }}<4250 \mathrm{~K}$ the situation seems more complex, but $\mathrm{CaHK}, \mathrm{Ca} 4227$, and $\mathrm{Mg} b$ remain among the most $\alpha$-sensitive, and G4300 and Ca4455 remain among the least $\alpha$-sensitive indices.

\section{RESULTS}

We caution that because our models have scaled-solar abundances, in the discussion that follows, the $[\mathrm{M} / \mathrm{H}]$ parameter is effectively identical to the $[\mathrm{Fe} / \mathrm{H}]$ parameter in the internal context of our modeling and analysis, whereas in

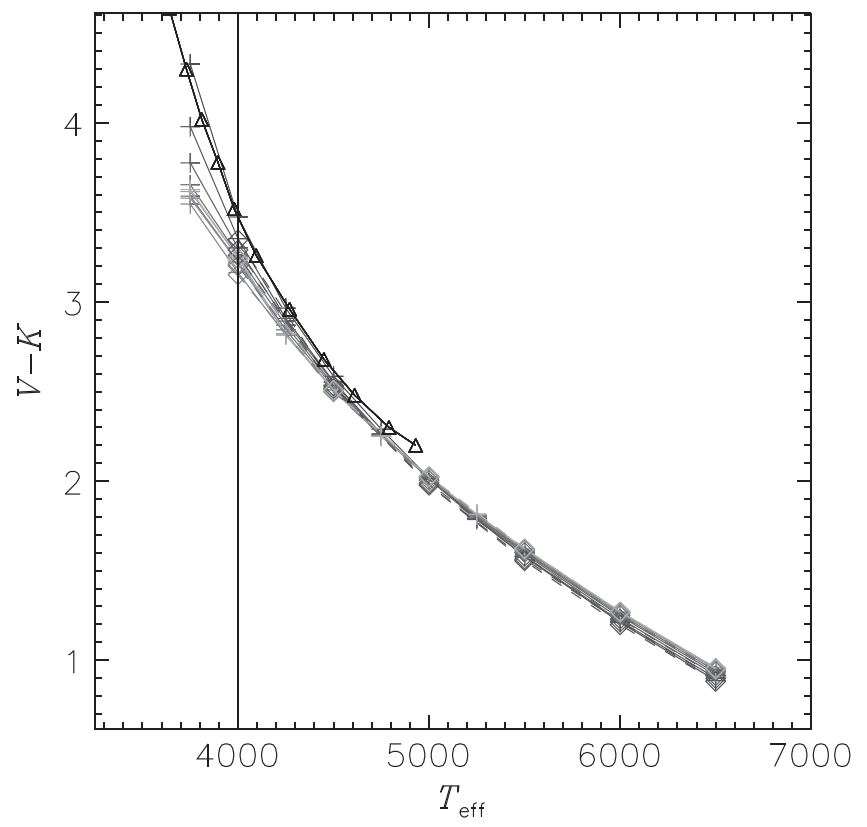

Figure 4. $V-K\left(T_{\text {eff }}\right)$ relation. Synthetic colors from LTE (solid grayscale lines and crosses) and NLTE (dashed grayscale lines and diamonds), and the calibration of Ridgway et al. (1980; solid black line and triangles). The synthetic colors were tied to the Ridgway et al. (1980) relation with a singlepoint calibration of the $[\mathrm{M} / \mathrm{H}]=0.0$ models at $T_{\text {eff }}=4000 \mathrm{~K}$ (vertical line) Grayscale: darker lines indicates larger $[\mathrm{M} / \mathrm{H}]$ values throughout the model grid range of -6.0 to 0.0 . Note that we only compute NLTE $I$ values at a subset of the LTE grid-see the text.

$\alpha$-enhanced metal-poor RGB stars with non-solar abundance distributions, $[\mathrm{M} / \mathrm{H}]$ differs from $[\mathrm{Fe} / \mathrm{H}]$. This distinction is expected to be most important for those Lick indices that are $\mathrm{Mg}$ - or Ca-dominated, and less so for those that are $\mathrm{Fe}$ dominated.

\section{1. $V-K\left(T_{\mathrm{eff}}\right)$ and $V-K([\mathrm{M} / \mathrm{H}])$ Relations}

Figure 4 shows the LTE and NLTE model $V-K\left(T_{\text {eff }}\right)$ relation for our range of model $[\mathrm{M} / \mathrm{H}]$ values, overplotted with the $V-K\left(T_{\text {eff }}\right)$ relation for giants of Ridgway et al. (1980). The model $V-K\left(T_{\text {eff }}\right)$ relation flattens with a decreasing $[\mathrm{M} / \mathrm{H}]$ value, which is to be expected because line blanketing extinction in the $V$ band increases more rapidly with an increasing $[\mathrm{M} / \mathrm{H}]$ value than that in the $K$ band. Within the range of overlap in $T_{\text {eff }}(3750-5000 \mathrm{~K})$, our model $V-K\left(T_{\text {eff }}\right)$ relation at $[\mathrm{M} / \mathrm{H}]=0.0$ closely tracks that of Ridgway et al. (1980), but is slightly steeper. However, the Ridgway et al. (1980) sample of red giants probably includes stars of $[\mathrm{Fe} / \mathrm{H}]<0.0$, so it should have a flatter $V-K\left(T_{\text {eff }}\right)$ relation than that of $[\mathrm{M} / \mathrm{H}]=0.0$. NLTE effects are negligible, which is to be expected given that these are broadband colors that average the effects of many spectral lines, and that line blanketing opacity is already considerably reduced in the $V$ band as compared to the $B$ and $U$ bands.

\subsection{XMP Indices}

Table 2 displays, for a selection of $T_{\text {eff }}$ values spanning our grid, the range of $[\mathrm{M} / \mathrm{H}]$ values for which each index, $I$, is a sensitive $[\mathrm{M} / \mathrm{H}]$ indicator as judged by the criterion that $\Delta[\mathrm{M} / \mathrm{H}] \times \frac{\partial I}{\partial[\mathrm{M} / \mathrm{H}]} \gtrsim \sigma_{\text {Worthey }}$, where $\Delta[\mathrm{M} / \mathrm{H}] \approx 1$ and $\sigma_{\text {Worthey }}$ is an observational uncertainty described below. Lick 
Table 2

Range of the $[\mathrm{M} / \mathrm{H}]$ Value for Which Lick Index, $I$, is a Significant $[\mathrm{M} / \mathrm{H}]$ Diagnostic for Differences of $\Delta[\mathrm{M} / \mathrm{H}] \approx 1$ for Select $T_{\mathrm{eff}}$ Values, and Corresponding $(V-K)$ Values at $[\mathrm{M} / \mathrm{H}]=0.0$, at SDSS Spectral Resolution

\begin{tabular}{|c|c|c|c|c|}
\hline \multirow[b]{4}{*}{ Index } & \multicolumn{4}{|c|}{$T_{\text {eff }}(\mathrm{K})$} \\
\hline & 3750 & 4500 & 5000 & 6500 \\
\hline & \multicolumn{4}{|c|}{$V-K$ at $[\mathrm{M} / \mathrm{H}]=0.0$} \\
\hline & 4.33 & 2.58 & 2.02 & 0.89 \\
\hline $\mathrm{CN}_{2}$ & $-5.0,-2.0$ & $-1.5,0.0$ & $-0.5,0.0$ & is \\
\hline $\mathrm{Ca} 4227$ & $-4.0,0.0$ & mv, neg & mv, neg & $-3.0,0.0$ \\
\hline G4300 & $\mathrm{mv}$ & $-3.0,-5.0$ & $-1.5,-4.0$ & $0.0,-3.0$ \\
\hline $\mathrm{Fe} 4383^{\mathrm{a}}$ & $-5.0,-1.0$ & $-4.0,0.0$ & $-4.0,-1.0$ & $-3.0,0.0$ \\
\hline $\mathrm{Ca} 4455$ & $-4.0,0.0$ & $-3.0,0.0$ & $-3.0,0.0$ & $-2.0,0.0$ \\
\hline $\mathrm{Fe} 4531^{\mathrm{a}}$ & $-5.0,0.0$ & $-4.0,0.0$ & $-3.0,0.0$ & $-3.0,0.0$ \\
\hline $\mathrm{Fe} 4668$ & $-1.0,0.0$ & $-1.5,0.0$ & $-1.5,0.0$ & $-1.0,0.0$ \\
\hline $\mathrm{Fe} 5015^{\mathrm{a}}$ & $-5.0,0.0$ & $-4.0,0.0$ & $-4.0,0.0$ & $-3.0,0.0$ \\
\hline $\mathrm{Mg}_{1}^{\mathrm{a}}$ & $-5.0,-2.0$ & $-3.0,0.0$ & $-2.0,0.0$ & $-1.0,0.0$ \\
\hline $\operatorname{Mg} b^{\mathrm{a}}$ & $-6.0,0.0$ & $-5.0,0.0$ & $\mathrm{mv}$ & $\mathrm{mv}$ \\
\hline $\mathrm{Fe} 5270^{\mathrm{a}}$ & $-6.0,0.0$ & $-4.0,0.0$ & $-3.0,0.0$ & $-2.0,0.0$ \\
\hline $\mathrm{Fe} 5335^{\mathrm{a}}$ & $-6.0,0.0$ & $-5.0,0.0$ & $-4.0,0.0$ & $-3.0,0.0$ \\
\hline $\mathrm{Fe} 5406^{\mathrm{a}}$ & $-6.0,0.0$ & $-5.0,0.0$ & $-3.0,0.0$ & $-2.0,0.0$ \\
\hline Fe5709 & $-4.0,0.0$ & $-3.0,0.0$ & $-3.0,0.0$ & $-1.0,0.0$ \\
\hline Fe5782 & $-3.0,-1.0$ & $-2.0,0.0$ & $-2.0,0.0$ & $-1.0,0.0$ \\
\hline $\mathrm{Na} D^{\mathrm{a}}$ & $-6.0,0.0$ & $-5.0,0.0$ & $-4.0,0.0$ & is \\
\hline $\mathrm{TiO}_{1}$ & $-3.0,0.0$ & $-1.0,0.0$ & $-1.0,0.0$ & is \\
\hline $\mathrm{TiO}_{2}$ & $-4.0,0.0$ & $-3.0,0.0$ & $-2.0,0.0$ & $-1.0,0.0$ \\
\hline
\end{tabular}

Notes. is, mv, neg: $I$ suffers from one or more of several pathologies over a significant range of $[\mathrm{M} / \mathrm{H}]$. is: $I$ is insensitive to $[\mathrm{M} / \mathrm{H}]$ as judged by the corresponding value of $\sigma_{\text {Worthey }}$ (see the text). mv: $I$ is multi-valued. neg: $I$ is negative (for those that are in linear $W_{\lambda}$ units).

${ }^{a} I$ is a strong, well-behaved $[\mathrm{M} / \mathrm{H}]$-indicator over a broad $[\mathrm{M} / \mathrm{H}]$ range down to XMP values (at least $[\mathrm{M} / \mathrm{H}] \leqslant-5.0$ ) for at least some GK star $T_{\text {eff }}$ values.

indices that meet this criterion and remain strong enough at VMP-to-XMP metallicities $([\mathrm{M} / \mathrm{H}]<-4.0)$ to be detectable are considered to be "Lick-XMP" indices. Generally, all of the "metallic" atomic and molecular indices have $\frac{\partial I}{\partial[\mathrm{M} / \mathrm{H}]}$ values that increase with a decreasing $T_{\text {eff }}$ value, and we expect that Lick-XMP indices will be more readily identifiable at the cool end of our grid.

Table 2 includes indications for indices that become "pathological" over a significant $[\mathrm{M} / \mathrm{H}]$ range at any $T_{\text {eff }}$ values by becoming multi-valued, or negative in the case of those indices that are in linear $W_{\lambda}$ units. These pathologies often reflect complications in the bracketing pseudo-continuum wavebands as defined by the Lick index standard rather than with the central feature itself and most often appear at higher $[\mathrm{M} / \mathrm{H}]$ values.

At $T_{\text {eff }}=3750 \mathrm{~K}$, we have found five Lick indices that meet our Lick-XMP criterion all the way down to $[\mathrm{M} / \mathrm{H}]=-6.0$ (Fe5270, Fe5335, Fe5406, Mg $b, \mathrm{Na} D$ ), and four others that meet the criterion down to $[\mathrm{M} / \mathrm{H}]=-5.0(\mathrm{Fe} 4383, \mathrm{Fe} 4531$, Fe5015, $\left.\mathrm{Mg}_{1}\right)$. At $T_{\text {eff }}=4500 \mathrm{~K}$ there are none that meet the criterion down to $[\mathrm{M} / \mathrm{H}]=-6.0$, and four of the above nine that still meet the criterion down to $[\mathrm{M} / \mathrm{H}]=-5.0(\mathrm{Fe} 5335$, Fe5406, $\mathrm{Mg} b$, Na $D$ ). These nine Lick-XMP indices are indicated in Figures 2 and 3 with identification labels that are off-set from the rest. By $T_{\text {eff }}=5000 \mathrm{~K}$ (and hotter) there are no indices that meet our Lick-XMP criterion. (Furthermore, we caution that $\mathrm{Mg} b$ becomes multi-valued as a function of $[\mathrm{M} / \mathrm{H}]$ for $T_{\text {eff }} \gtrsim 5000 \mathrm{~K}$.) The presence of Fe4383, Fe5270, and Fe5335 among our Lick-XMP indices is not surprising given that Gorgas et al. (1993) identified them as strong [Fe/H] -indicators. $\mathrm{Na} D$ is an interesting member of our Lick-XMP indices in that it is not Fe-dominated, but we caution, again, that its usefulness is compromised by significant ISM extinction. As noted in Section 2, our treatment of $\mathrm{Mg} b$ and $\mathrm{Mg}_{1}$ is least accurate because we neglect $\alpha$-enhancement in the current investigation, and $\mathrm{Mg}$ is an $\alpha$-process element. Therefore, the following results for these two indices are most suspect and require further investigation.

Figures 5-8 show the modeled $I([\mathrm{M} / \mathrm{H}])$ relation at $T_{\text {eff }}=4000 \mathrm{~K}$, and Figures 9-12 show the modeled $I\left(T_{\text {eff }}\right)$ relation at $[\mathrm{M} / \mathrm{H}]=0.0$ for four of our Lick-XMP indices, Fe5270, Fe4383, $\mathrm{Mg} b$, and $\mathrm{Na} D$. The latter are the only two Lick-XMP indices not dominated by Fe. Results are shown for spectra computed with the $R$ values of SDSS and IDS spectroscopy, as computed in LTE and NLTE. For comparison, we have overplotted observationally derived $I\left(T_{\text {eff }}\right)$ and $I([\mathrm{M} / \mathrm{H}])$ points for a wide range of cool giants from the catalog of Worthey \& Ottaviani (1997) with catalog $\log g$ values between 1.0 and 3.0 , and $T_{\text {eff }}$ and $[\mathrm{M} / \mathrm{H}]$ within $\pm 100 \mathrm{~K}$ and \pm 1.0 , respectively, of the plotted models. Note that the Worthey \& Ottaviani (1997) I data only includes stars of $[\mathrm{M} / \mathrm{H}] \gtrsim-1.0$, and that $n o$ calibration of our $I$ values to those of Worthey \& Ottaviani (1997) has been performed. Nevertheless, from Figures 7 and 8, the agreement between our modeled $\mathrm{Mg} b$ and $\mathrm{Na} D I$ values and the measurements of Worthey \& Ottaviani (1997) for stars of $T_{\text {eff }}=4000 \pm 100 \mathrm{~K}$ is assuring. Figures 5-8 show that these indices generally satisfy our Lick-XMP criteria for $T_{\text {eff }}$ values in the cool part of our grid. Figure 10 shows that the utility of Fe4383 is somewhat compromised-it is double-valued as a function of $T_{\text {eff }}$ at the cool edge of our grid. However, it is a sensitive and useful $[\mathrm{M} / \mathrm{H}]$ discriminator otherwise, and we include it because there are few indices that qualify as Lick-XMP at all. Figures 9-12 show that, as discussed above, Lick-XMP indices quickly become weaker and lose their ability to discriminate among $[\mathrm{M} / \mathrm{H}]$ values for $T_{\text {eff }} \gtrsim 5000 \mathrm{~K}$. Figures 5 through 12 also include an indication of the "observational uncertainty" as determined by W94, $\sigma_{\text {Worthey }}$ (see below), to aid in assessing the significance of $\Delta I$ differences.

The effect of NLTE is complex in that every Lick index is really a compound feature caused by significant spectral lines from several species. Moreover, although the bracketing pseudo-continua used to define the indices were chosen to be relatively insensitive to stellar parameter values, NLTE effects on line strengths in the bracketing regions will, in principle, also play a role in the overall effect of NLTE on the computed $I$ value. However, Fe5270 is typical of our results for the Fedominated indices in that the effect of the well-known NLTE over-ionization of $\mathrm{Fe}_{\mathrm{I}}$ in late-type stars (Rutten 1986) leads to smaller $I$ values at every $T_{\text {eff }}-[\mathrm{M} / \mathrm{H}]$ combination. Figures 13 and 14 show the size of the NLTE effect, $\Delta I \equiv I_{\mathrm{NLTE}}-I_{\mathrm{LTE}}$, as a function of $T_{\text {eff }}$ for Fe5270 and $\mathrm{Mg} b$. The effect of NTLE on strong low $\chi \mathrm{Fe}_{\mathrm{I}}$ lines is to weaken them (a negative correction to modeled I value) as a result of NLTE overionization. In late-type stars, the effect will generally be maximal where the discrepancy, $\Delta T$, throughout the line forming region between the radiation temperature, $T_{\mathrm{Rad}}$, of the photo-ionizing near-UV band radiation in the NLTE treatment, and the local kinetic temperature, $T_{\mathrm{Kin}}$, that determines the ionization balance in the LTE treatment, is largest. For Fe $\mathrm{I}$ in giants, the discrepancy is largest around $T_{\mathrm{eff}} \approx 5000 \mathrm{~K}$, and 


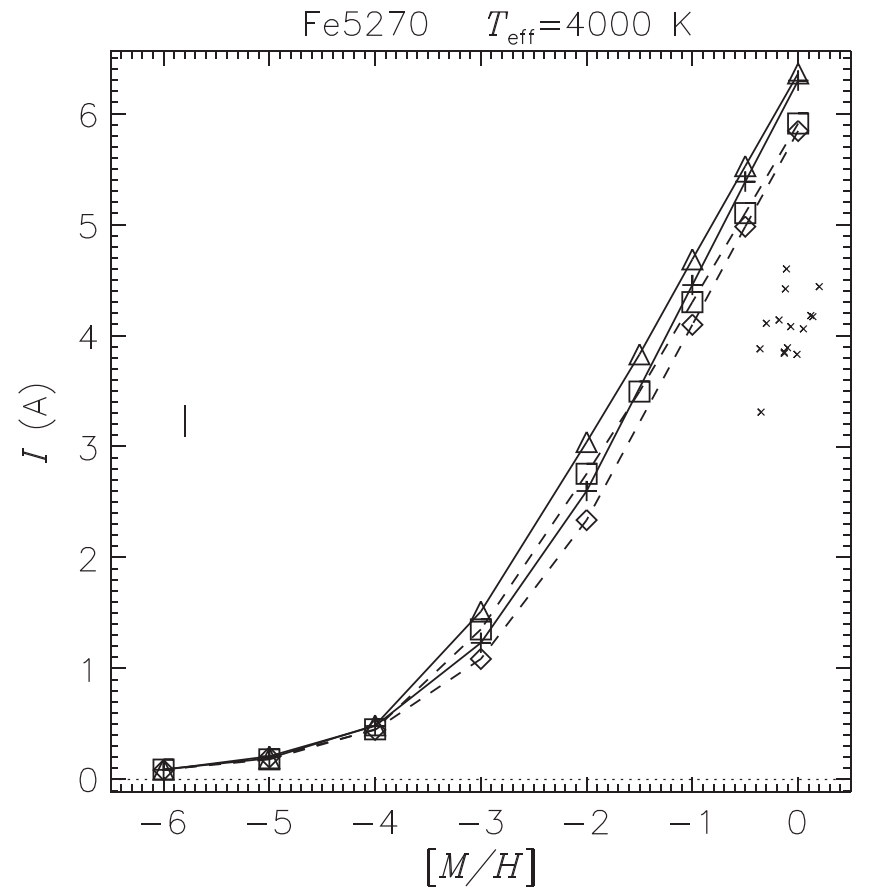

Figure 5. Index Fe5270, one of our identified Lick-XMP indices: $I$ as a function of $[\mathrm{M} / \mathrm{H}]$ at $T_{\text {eff }}=4000 \mathrm{~K}$. Vertical line in middle left: Indication of the observational uncertainty, $\sigma_{\text {Worthey }}$, of W94-see the text. $I$ has been computed from spectra with $R$ values of SDSS (solid line) and IDS (dashed line) spectroscopy that were computed in LTE (triangles, squares) and NLTE (crosses, diamonds) - see the text. The vertical spread in $I$ values at a given $[\mathrm{M} / \mathrm{H}]$ value can be taken as an estimate of "spectroscopic and modeling physics uncertainty." Note that we only compute NLTE $I$ values at a subset of the LTE grid - see the text. For comparison we show the observationally derived $I$ and $T_{\text {eff }}$ values of Worthey \& Ottaviani (1997) for giants $\left(1.0 \leqslant \log g \leqslant 3.0\right.$ ) of $T_{\text {eff }}$ within $\pm 100 \mathrm{~K}$ ("X" symbols). No calibration of our $I$ values to those of Worthey \& Ottaviani (1997) has been performed.

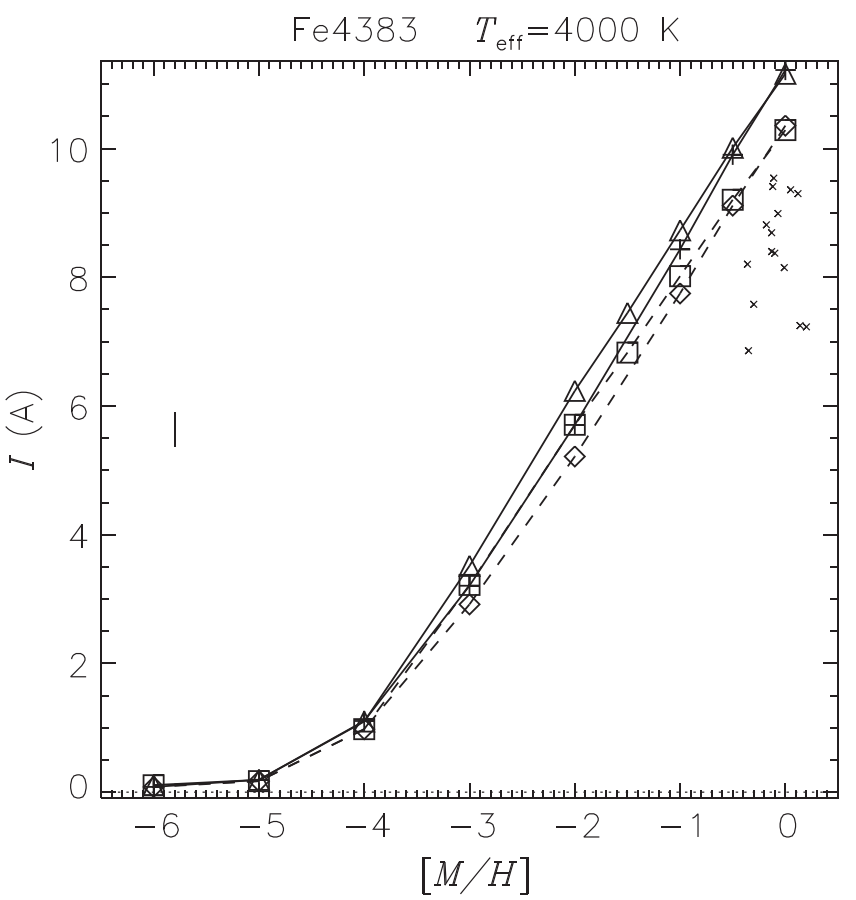

Figure 6. Same as Figure 5, but for index Fe4383.

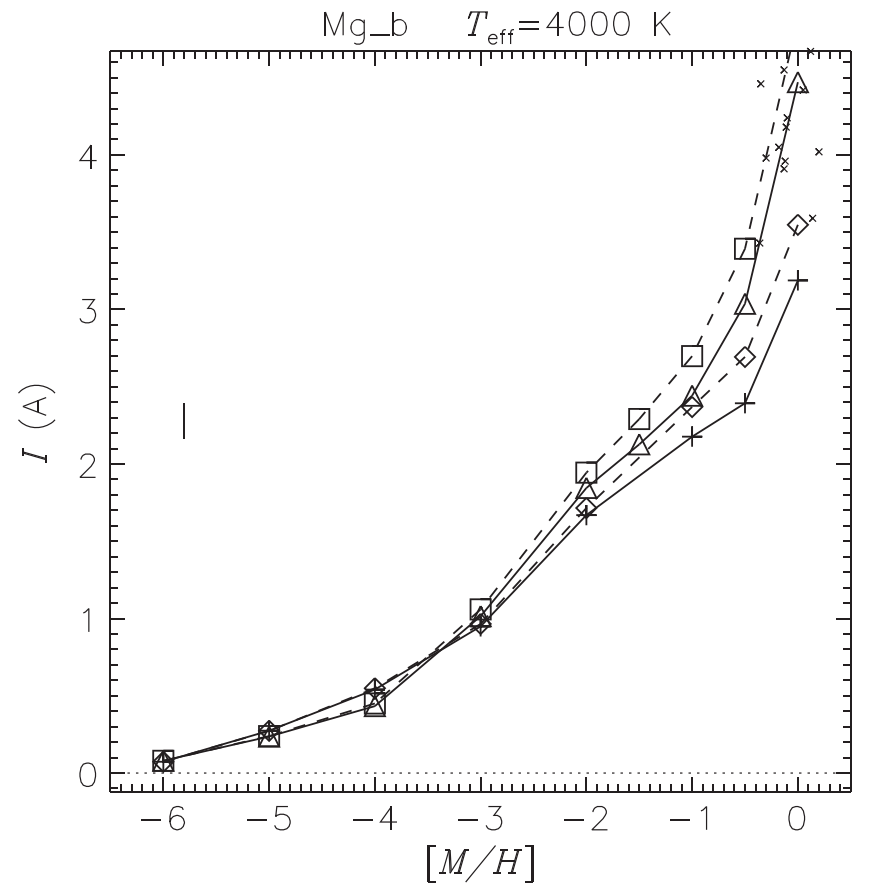

Figure 7. Same as Figure 5, but for index $\operatorname{Mg} b$, an example of one of our identified Lick-XMP indices, not dominated by Fe.

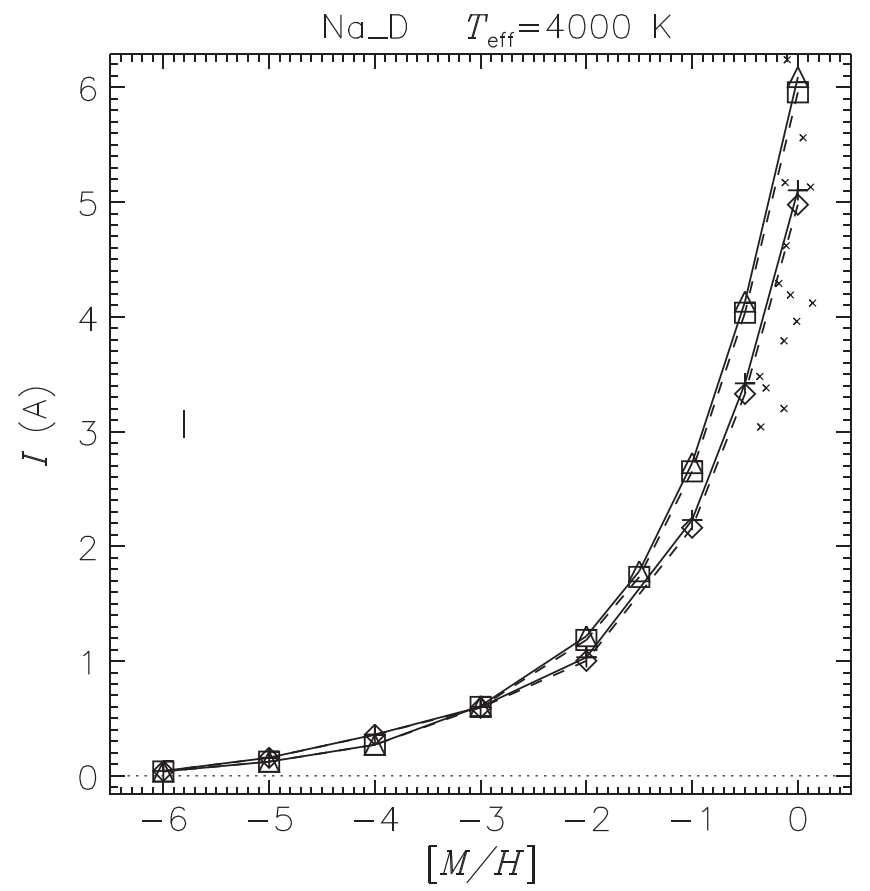

Figure 8. Same as Figure 5, but for index Na D, another example of one of our identified Lick-XMP indices, not dominated by Fe.

decreases in magnitude for both lower and higher $T_{\text {eff }}$ values (see Rutten 1986 for a thorough analysis for the case of $\mathrm{Fe}$ in the Sun).

For $\mathrm{Mg}$ I (Index $\mathrm{Mg} b$ ) the NLTE correction to the modeled $I$ value is also negative, but increases in magnitude with decreasing $T_{\text {eff }}$ throughout this $T_{\text {eff }}$ range, especially for $T_{\text {eff }}<4000 \mathrm{~K}$. Osorio et al. (2015) very recently conducted a thorough NLTE analysis of $\mathrm{Mg}$ I in late-type stellar atmospheres, including an investigation of $\mathrm{H}$ I collisional cross- 


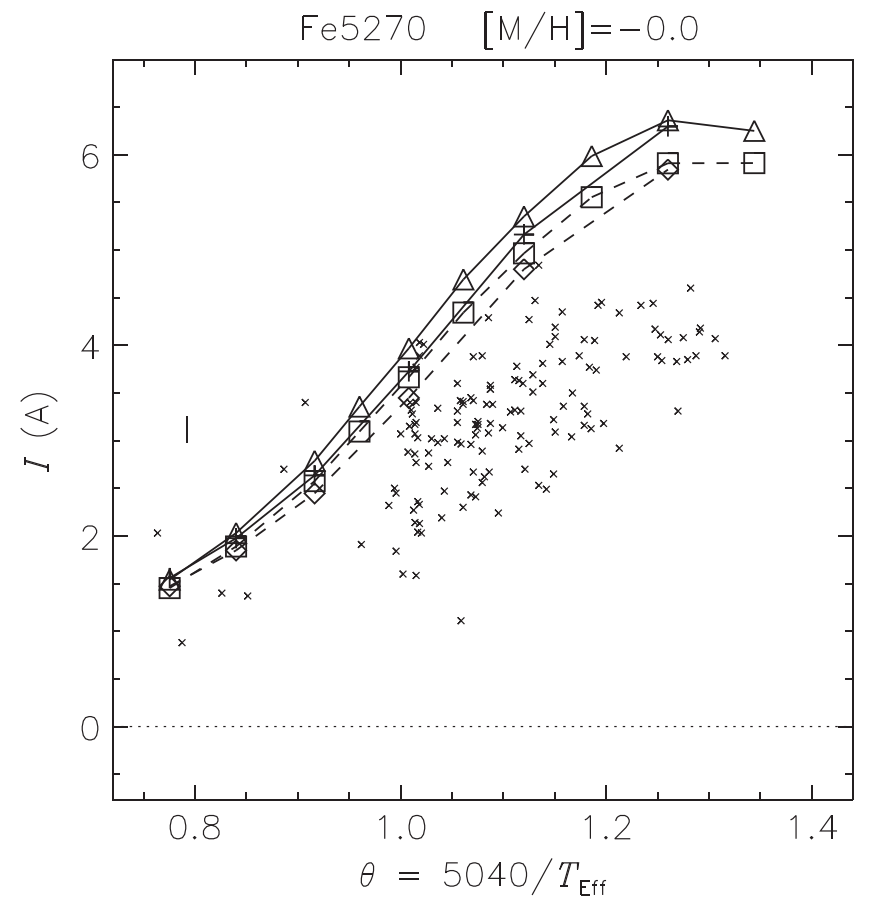

Figure 9. Same as Figure 5, but for $I$ as a function of $\theta=5040 / T_{\text {eff }}$ at $[\mathrm{M} / \mathrm{H}]=0.0$. For comparison, we show the observationally derived $I$ and $[\mathrm{M} / \mathrm{H}]$ values of Worthey \& Ottaviani (1997) for stars of $[\mathrm{M} / \mathrm{H}]$ within \pm 1.0 ("X" symbols).

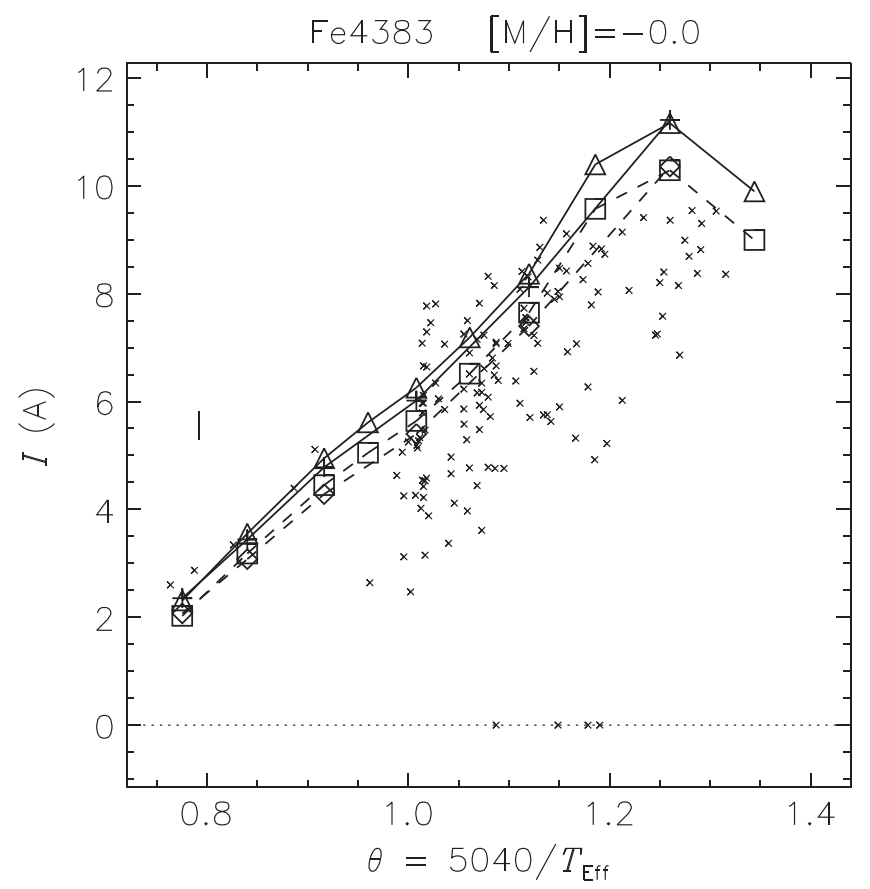

Figure 10. Same as Figure 9, but for index Fe4383. $I$ is double valued at the cool edge of the grid, making its use there problematic, but is generally useful as an $[\mathrm{M} / \mathrm{H}]$ diagnostic otherwise.

sections and electron-exchange reactions. For the $\lambda 5184$ line, they found that NLTE effects lead to a weakening of the modeled line (hence the positive abundance correction in their Figure 10), by an amount that depends on choice of atomic data, but can be as much as 0.4 dex at $T_{\text {eff }}=4500 \mathrm{~K}$, $\log g=1.0$, and $[\mathrm{M} / \mathrm{H}]=0.0$, which is qualitatively consistent with our result.

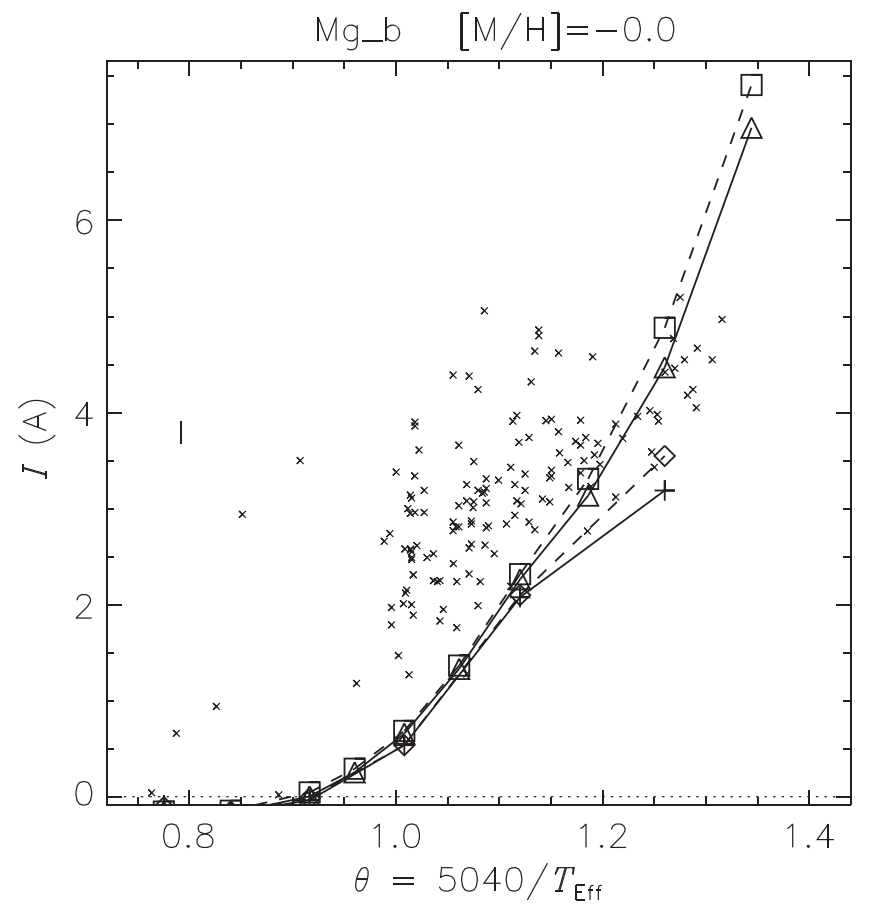

Figure 11. Same as Figure 9, but for index $\operatorname{Mg} b$.

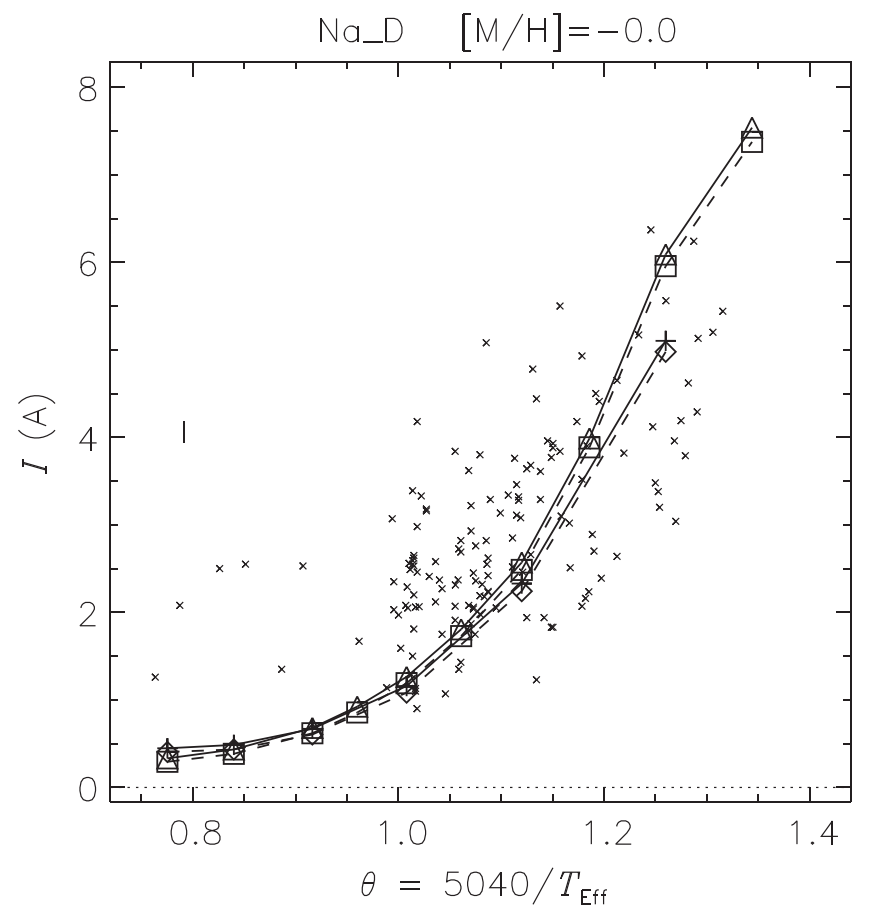

Figure 12. Same as Figure 9, but for index Na $D$.

Interestingly, the magnitude of NLTE effect on the computed $I$ value is comparable to that caused by changing spectral resolution ( $R$ value of IDS versus SDSS). Na $D$ is an exception because it is so broad that it is minimally affected by the choice of $R$. Computing $I$ from higher $R$-value spectra (i.e., that of SDSS) can either increase or decrease the $I$ value, depending on the index. The same remarks as made when considering NLTE effects above also apply: namely, that the effect of $R$ value on any given index will depend on how the bracketing pseudo-continua are affected as well as the central 


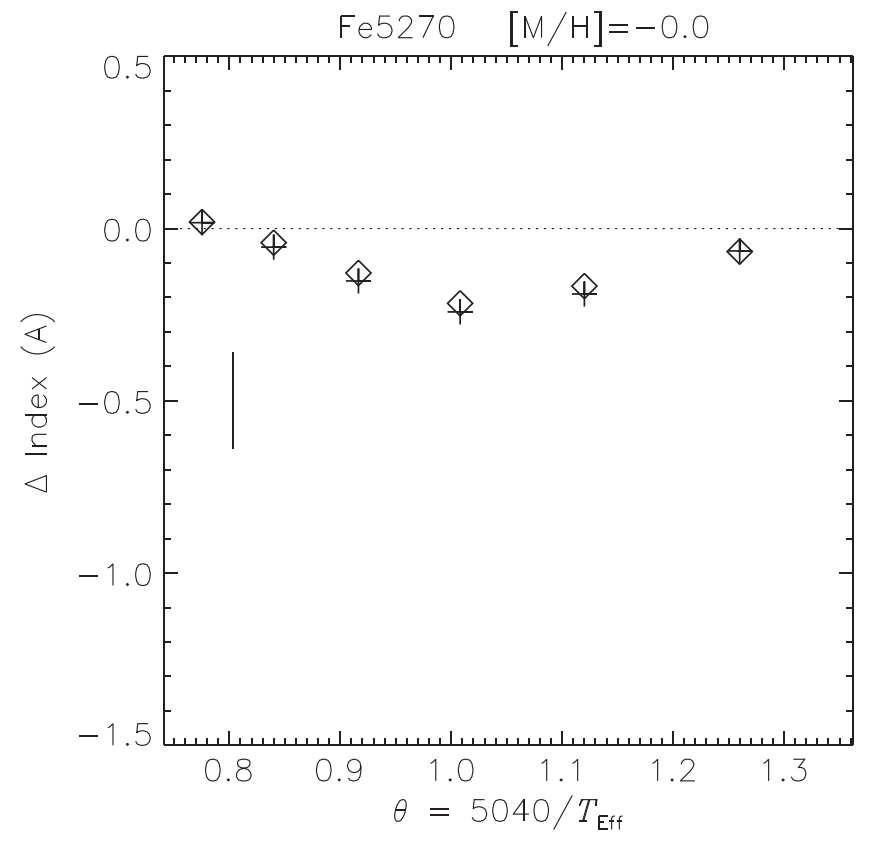

Figure 13. Index Fe5270: the difference between $I$ computed in NLTE and that in LTE, $\Delta I=I_{\mathrm{NLTE}}-I_{\mathrm{LTE}}$, as a function of $\theta=\frac{5040}{T_{\text {eff }}}$ for $[\mathrm{M} / \mathrm{H}]=0.0$. Results for $I$ derived from spectra of SDSS (crosses) and IDS (diamonds) resolution, $R$. The horizontal dotted line indicates a $\Delta I$ value of zero.

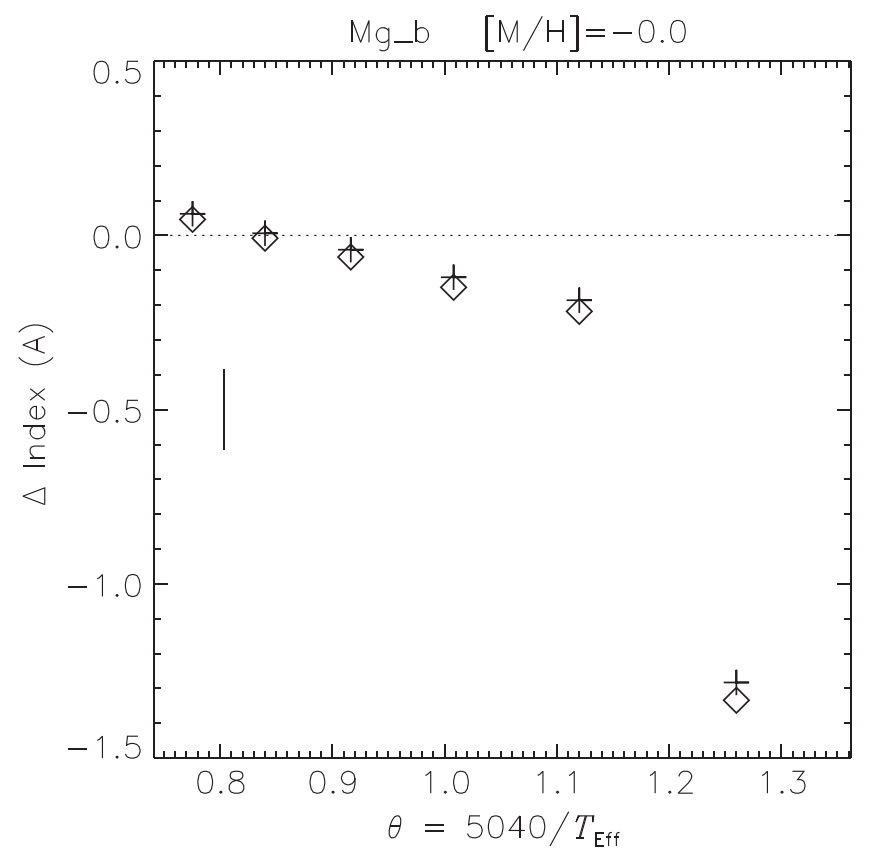

Figure 14. Same as Figure 13, except for index $\operatorname{Mg} b$.

feature itself. Altogether, the vertical spread in $I$ values at each abscissa can be taken as an approximate indication of "spectroscopic and modeling physics uncertainty."

\subsection{Polynomial Fits}

Based on the approach taken by W94 with observed IDS spectra and observationally determined stellar parameters, we have used multi-variate linear regression to determine 10 polynomial fitting (regression) coefficients (or model parameters), $C_{\mathrm{n}}$, for each index, $I$, for a polynomial fitting function,
$P_{3}$, that accounts for all terms, including cross-products, up to third order in $[\mathrm{M} / \mathrm{H}]$ and $\log \theta$, where $\theta \equiv 5040 / T_{\text {eff }}$,

$$
\begin{aligned}
I \approx P_{3} \equiv & C_{0} \\
& +C_{1}[\mathrm{M} / \mathrm{H}]+C_{2} \log \theta+C_{3}[\mathrm{M} / \mathrm{H}]^{2} \\
& +C_{4} \log ^{2} \theta+C_{5}[\mathrm{M} / \mathrm{H}] \log \theta \\
& +C_{6}[\mathrm{M} / \mathrm{H}]^{3}+C_{7} \log ^{3} \theta \\
& +C_{8}[\mathrm{M} / \mathrm{H}]^{2} \log \theta+C_{9}[\mathrm{M} / \mathrm{H}] \log ^{2} \theta .
\end{aligned}
$$

Note that, as per convention, the units of $I$ for the molecular indices $\mathrm{CN}_{1}, \mathrm{CN}_{2}, \mathrm{Mg}_{1}, \mathrm{Mg} 2, \mathrm{TiO}_{1}$, and $\mathrm{TiO}_{2}$ are magnitudes, and those for the remaining indices are $\AA$. Moreover, following W94, in the special case of the $\mathrm{TiO}_{1}$ and $\mathrm{TiO}_{2}$ indices, which exhibit a rapid increase in strength with decreasing $T_{\text {eff }}$ near the lower limit of our $T_{\text {eff }}$ range, we fit Equation (1) to $\log I$ so that $I$ is being fit by $\exp P_{3}$. The fitting is performed with the intrinsic REGRESS procedure in Interactive Data Language (IDL) and is achieved by minimizing the $\chi^{2}$ figure of merit assuming that the uncertainty, $\sigma_{\mathrm{i}}(I)$, of all "data" points $I_{\mathrm{i}}$, is unity. We note that IDL installations include the source code for all intrinsic procedures, and we have been able to critically inspect the REGRESS procedure. We use REGRESS to compute nine linear regression coefficients and a constant term in Equation (1) for nine basis functions that consist of the powers of the independent parameters $\left(T_{\text {eff }},[\mathrm{M} / \mathrm{H}]\right)$ and their products up to third order. This amounts to fitting a model with nine parameters to 90 data points (90 computed $I$ values for ten $T_{\text {eff }}$ and nine $[\mathrm{M} / \mathrm{H}]$ values), thus having 81 degrees of freedom. This is consistent with the fitting method of W94-we note that their Equation (4) appears to be the standard formula for $\chi^{2}$ because their summed square deviations are weighted by their inverse observational uncertainty, $1 / \sigma^{2}$, although they have labeled their figure of merit " $\mathrm{rms}^{2}$." We note that because we have no proper data uncertainties (i.e., "measurement errors" in data modeling), $\sigma_{\mathrm{i}}(I)$, we cannot properly propagate errors to compute uncertainties for the fitted model parameters, $C_{\mathrm{n}}-$ rather we compute "fitting uncertainties," $\sigma$, post hoc from the calculated value of $\chi^{2}$ and the number of degrees of freedom. Assuming these "fitting uncertainties" reflect errors that are normally distributed, they may be interpreted as $68 \%$ confidence intervals for the fitted values of the corresponding $C_{\text {n. }}$.

Tables 3 and 4 present these $C_{\mathrm{n}}$ values for LTE spectra of IDS and SDSS resolution, respectively, for the nine indices that were identified above as good Lick-XMP diagnostics, in the same format and numerical precision as that of Table 2 of W94 ("Data for stars of $3570<T_{\text {eff }}<5160 \mathrm{~K}$ ") for direct comparison. Table 5 presents the results for IDS spectral resolution of performing the same third order multiple linear regression with the model $V-K$ color in place of $\log \theta$. We also present the values of the standard deviations, $\sigma$, computed for each $C_{\mathrm{n}}$ parameter from $\chi^{2}$, and the value of the reduced $\chi^{2}$ given 81 degrees of freedom, although we caution that in the absence of proper measurement errors, $\chi^{2}$ is not really a goodness-of-fit figure of merit. Figures 15 through 18 show the comparison of the polynomial fits to the modeled $I\left(T_{\text {eff }}\right)$ relation for $[\mathrm{M} / \mathrm{H}]=0.0$ at SDSS and IDS resolution, and the residuals. For SDSS resolution, we also show both the fitted relation and the residuals computed with the $1 \sigma$ "fitting uncertainties" (see above) added and subtracted from each of the $C_{\mathrm{n}}$ values to 
illustrate these two limiting cases. Figures 19 through 22 present the same information for the polynomial fits to the modeled $I([\mathrm{M} / \mathrm{H}])$ relation for $T_{\text {eff }}=4000 \mathrm{~K}$.

There are a number of important differences between our approach and that of W94.

a. Our $C_{\mathrm{n}}$ values complement those of W94 by being based on spectra of SDSS resolution rather than those of IDS resolution, and are more relevant to both SDSS and LAMOST spectra and to the investigation of Franchini et al. (2010).

b. $\log g$ is not a fitting parameter, so we do not have crossproduct terms that capture the dependence of $I$ on the product of $\log g$ or any of its powers and other parameters or their powers.

c. Table 2 of W94 contains coefficients for fits in the $T_{\text {eff }}$ range of $3570-5160 \mathrm{~K}$, whereas our fits apply to the range of $3750-6500 \mathrm{~K}$. The difference at the high $T_{\text {eff }}$ end is necessary for us to accommodate the $T_{\text {eff }}$ range of interest for detected halo red giants. Because the lower limit of our $T_{\text {eff }}$ range is significantly higher than that of W94, results for our TiO indices are especially suspect, and not comparable to W94. W94 included stars of $T_{\text {eff }}>5160 \mathrm{~K}$ in their fits for warm and hot stars (5040-13260 K, their Table 3). This is well beyond the limits of our red giant grid and we are not able to compare to their "hot star" fits.

d. W94 carry out a careful statistical $F$-value test of the goodness-of-fit to separately determine whether the addition of each successive $C_{\mathrm{n}}$ term in Equation (1) led to a statistically significant change in the fitted $I$ value for each index. As a result, many of the $C_{\mathrm{n}}$ values in their Table 2 are blank because, presumably, including the corresponding term in the fitted function led to an insignificant reduction in the variance. We have chosen to simply let the regression find $C_{\mathrm{n}}$ values for all 10 terms in Equation (1) consistently for all indices, with the expectation that terms that are of low significance will have small fitted values of the corresponding $C_{\mathrm{n}}$. Our expectation is that a third order fit is of a low enough order that we do not expect high order spurious solutions to compromise the fit, and our situation is simpler than that of W94 in that $\log g$ is not a fitting parameter.

Error analysis: W94 describes the uncertainty in determining an $I$ value as a "typical rms error per observation," which we denote $\sigma_{\text {Worthey. }}$. For reference, we have included an indicator of the magnitude of $\sigma_{\text {Worthey }}$ for each index in Figures 5 through 12. W94 quantifies the uncertainties in the fitted $I$ values with a "residual" rms value in units of the observational uncertainty, $\sigma_{\text {Worthey. }}$ We are not working with observational data, and we quantify the uncertainties in our fits, $\sigma$, with the quadrature sum of the $1 \sigma$ uncertainty estimates computed for each $C_{\mathrm{n}}$ value from the multiple linear regression procedure (as described above), and have included them in Tables 3 through 5 . Generally, the first order coefficients, $C_{1}$ for $[\mathrm{M} / \mathrm{H}]$ and $C_{2}$ for $T_{\text {eff }}$, from the fit to SDSS resolution spectra are larger than those from the fit to the IDS resolution spectra. This is to be expected because, in spectra of higher $R$ values, the first order dependence of the strength of spectral features on stellar parameters is less diluted by the reshuffling of information within the instrumental spectral profile.
The coefficient of the $\log ^{3} \theta$ term, $C_{7}$, generally had, by far, the largest $1 \sigma$ uncertainty value of all the $C_{\mathrm{n}}$ values, and dominates our quadrature sum $\sigma$ values. Generally, the parameter with next largest $1 \sigma$ uncertainty was the $\log ^{2} \theta$ term, $C_{4}$, but it was much smaller than that of $C_{7}$. Table 3 shows that the magnitude of $C_{7}$ is generally larger than any of the other coefficients, and this is consistent with what was reported in Table 2 of W94. We also found that the value of $C_{7}$ was the most sensitive to spectral resolution, differing by as much as a factor of four between the fits to IDS and SDSS resolution spectra. We conclude that the terms in nonlinear powers of $\log \theta$ were generally the least well fit. However, for these GK stars, $\theta \equiv 5040 / T_{\text {eff }}$ is of the order of unity, and the squares and cubes of the independent variable $\log \theta$ is much less than unity. We have found that the $1 \sigma$ uncertainty of the $C_{4}$ and $C_{7}$ terms for the analogous $V-K^{2}$ and $V-K^{3}$ terms from the fits with $V-K$ in lieu of $T_{\text {eff }}$ are much smaller and are consistent with those of the other $C_{\mathrm{n}}$ coefficients, and from Table 5 it can be seen that the corresponding total $\sigma$ values are smaller.

For our nine XMP indices, we compare our fitted $C_{\mathrm{n}}$ values for the zeroth and first order terms of the fit to IDS resolution spectra with those of W94, given the four caveats listed above. For the fits with $\log \theta$ as an independent parameter, comparable to Table 2 of W94, for five of six indices designated "Fe," our fitted $C_{0}$ value is consistently smaller than that of W94, and ranges from about $0.6-0.75$ of the W94 value, and for Fe5270 we are in very close agreement. For $\mathrm{Na} D$, our $C_{0}$ value is also smaller, but is in closer agreement with that of W94. $\mathrm{Mg}_{1}$ is our only index for which our $C_{0}$ is larger than W94, by about a factor of 1.5 . For $\mathrm{Mg} b$, we find a negative value of $C_{0}$, where W94 finds a positive value. In both cases, the magnitude of $C_{0}$ is about unity. For the $\log \theta$ coefficient, $C_{2}$, our values for Fe4531, Fe5335, and Fe5406 are close to the values of W94. The remaining $C_{2}$ values are also generally within a factor of two, greater or less than, and of the same sign as those of W94. The two exceptions are $\mathrm{Na} D$ for which our $C_{2}$ value is about three times larger, and $\mathrm{Mg}_{1}$ for which our $C_{2}$ value is positive and that of W94 is negative, although the magnitudes are within a factor of two. For the $[\mathrm{M} / \mathrm{H}]$ coefficient, $C_{1}$, our values for Fe5335, Fe5406, $\mathrm{Mg} b$, and $\mathrm{Mg}_{1}$ agree closely with those of W94. For the remaining XMP-Lick indices, including $\mathrm{Na} D$, our $C_{1}$ values differ by as much as a factor of 2.5 greater or less than those of W94.

For the fits with $V-K$ as an independent parameter (in lieu of $\log \theta$ ), only four of our nine Lick-XMP indices appear in the comparable Table 4 of W94 (Fe4383, Fe4531, Fe5015, and Fe5406). For Fe5015, W94 does not present a $C_{0}$ value, but our $C_{2}(I(V-K))$ agrees closely with theirs. For Fe4531, W94 does not present a $C_{2}$ value, and our $C_{0}$ value is about a factor of two larger. For both of these indices, our $C_{1}$ values $(I([\mathrm{M} / \mathrm{H}])$, at constant $V-K$ this time $)$ are close to those of W94. For Fe4383 and Fe5406, the situation is disconcerting and puzzling-we find both $C_{0}$ and $C_{2}$ values with the opposite sign and a difference in magnitude ranging from two to four. For both of these indices, W94 have no $C_{1}$ value for the term in $[\mathrm{M} / \mathrm{H}]$, indicating, presumably, that they found insignificant linear dependence of $I$ on $[\mathrm{M} / \mathrm{H}]$ at fixed $V-K$, and, consistently, we find modest $C_{1}$ values of -0.4489 and -0.0986 , respectively. We have been unable to identify the reason for the gross discrepancy in fitted polynomial coefficients for Fe4383 and Fe5406, beyond those expressed in the caveats itemized above. 
Table 3

LTE Fitting Function Coefficients, $C_{\mathrm{n}}$, for Independent Variables $\log \theta$ and $[\mathrm{M} / \mathrm{H}]$ for Stars of $3750<T_{\text {eff }}<6500 \mathrm{~K}$ at IDS Spectral Resolution for Our Nine LickXMP Indices

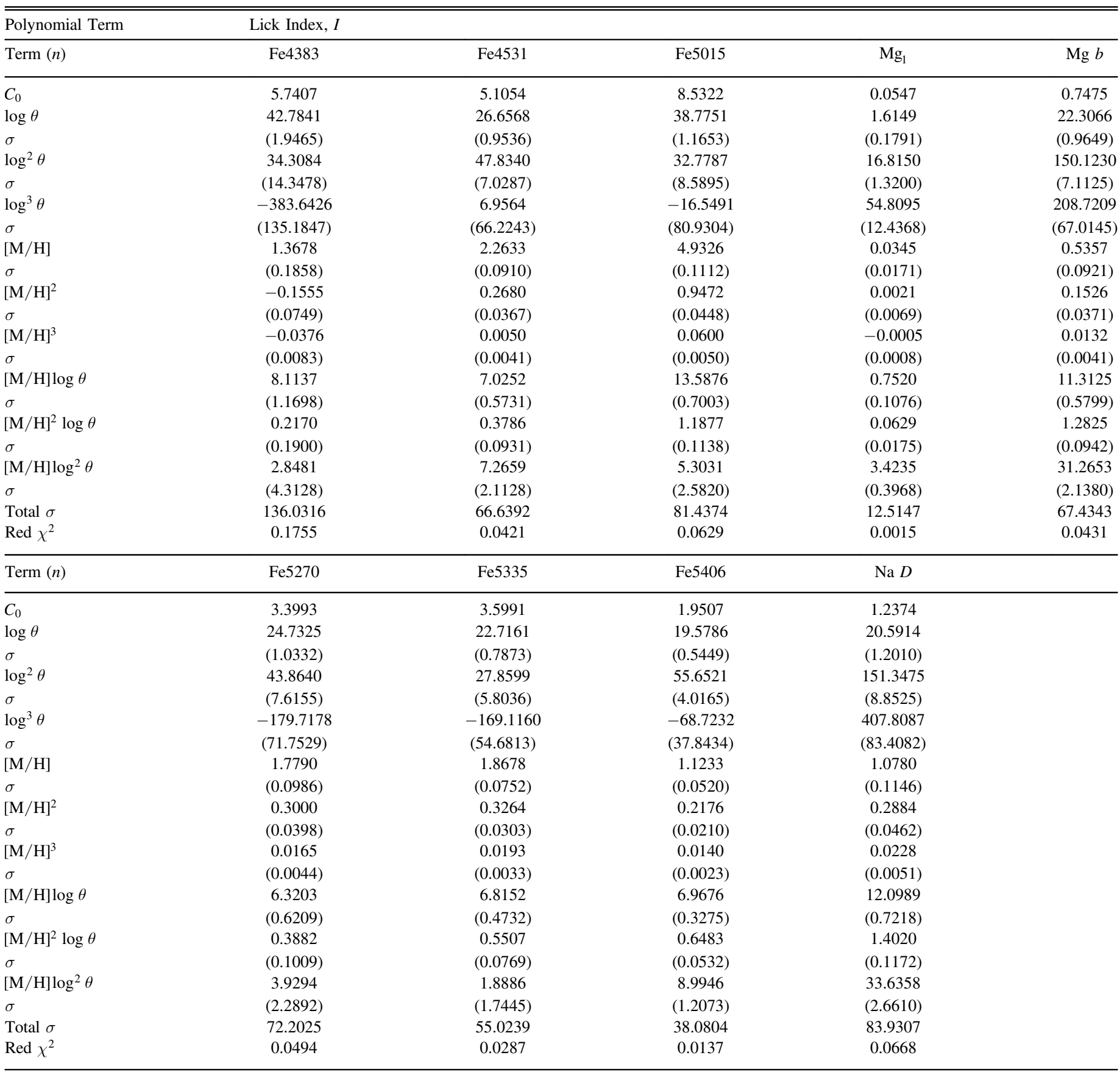

Note. Quantities in brackets below each fitted $C_{\mathrm{n}}$ value are the $1 \sigma$ "fitting errors" (see the text) estimated from $\chi^{2}$, and may be interpreted as $68 \%$ confidence intervals.

\subsection{Special Indices}

CaHK: Serven et al. (2005) introduced a new Lick-type index, which they designate CaHK, and Franchini et al. (2010) modified the definition by changing the blue pseudo-continuum band-pass and removing the central $5 \AA$ around the line cores to remove potential chromospheric emission. Franchini et al. (2010) found that CaHK had the advantage of being sensitive to $\alpha$-enhancement, as well as being less influenced by $\mathrm{Fe}$ than most of the other atomic indices. The index seems to show promise as a Lick-XMP $[\mathrm{M} / \mathrm{H}]$ diagnostic because the line is very strong. We do not remove the central $5 \AA$ from our index computation because our models have outer atmospheres that are in radiative equilibrium and do not have chromospheric emission. We have confirmed that we can reproduce the qualitative double-valued behavior of $I(\theta)$ exhibited in Figure 7 of Franchini et al. (2010). We have found that the index is a good Lick-XMP diagnostic for our models at the cool edge of our grid, of a $T_{\text {eff }}$ value equal to $3750-4000 \mathrm{~K}$. However, 
Table 4

Same as Table 3, but for Spectra of SDSS Spectral Resolution

\begin{tabular}{|c|c|c|c|c|c|}
\hline Polynomial Term & Lick Index, $I$ & & & & \\
\hline Term $(n)$ & $\mathrm{Fe} 4383$ & Fe4531 & Fe5015 & $\mathrm{Mg}_{1}$ & $\operatorname{Mg} b$ \\
\hline$C_{0}$ & 6.3207 & 5.8713 & 9.9981 & 0.0596 & 0.7159 \\
\hline $\log \theta$ & 46.5420 & 29.7759 & 47.4312 & 1.6445 & 21.1327 \\
\hline$\sigma$ & $(2.1429)$ & (1.1107) & (1.0168) & $(0.1790)$ & (1.0098) \\
\hline $\log ^{2} \theta$ & 37.7593 & 44.8405 & 37.9836 & 16.8348 & 136.5346 \\
\hline$\sigma$ & (15.7958) & $(8.1873)$ & $(7.4950)$ & (1.3194) & (7.4430) \\
\hline $\log ^{3} \theta$ & -426.9500 & -45.0185 & -124.2286 & 54.8555 & 149.0733 \\
\hline$\sigma$ & (148.8280) & (77.1404) & $(70.6182)$ & $(12.4315)$ & (70.1279) \\
\hline$[\mathrm{M} / \mathrm{H}]$ & 1.5700 & 2.5478 & 5.5798 & 0.0376 & 0.5076 \\
\hline$\sigma$ & $(0.2046)$ & $(0.1060)$ & $(0.0971)$ & $(0.0171)$ & $(0.0964)$ \\
\hline$[\mathrm{M} / \mathrm{H}]^{2}$ & -0.1475 & 0.2888 & 1.0275 & 0.0028 & 0.1489 \\
\hline$\sigma$ & $(0.0825)$ & $(0.0427)$ & $(0.0391)$ & $(0.0069)$ & $(0.0389)$ \\
\hline$[\mathrm{M} / \mathrm{H}]^{3}$ & -0.0392 & 0.0041 & 0.0624 & -0.0004 & 0.0134 \\
\hline$\sigma$ & $(0.0091)$ & $(0.0047)$ & $(0.0043)$ & $(0.0008)$ & $(0.0043)$ \\
\hline$[\mathrm{M} / \mathrm{H}] \log \theta$ & 8.5967 & 7.4703 & 15.1617 & 0.7638 & 10.7092 \\
\hline$\sigma$ & $(1.2879)$ & $(0.6675)$ & $(0.6111)$ & $(0.1076)$ & $(0.6068)$ \\
\hline$[\mathrm{M} / \mathrm{H}]^{2} \log \theta$ & 0.1880 & 0.3720 & 1.2213 & 0.0641 & 1.2324 \\
\hline$\sigma$ & $(0.2092)$ & $(0.1084)$ & $(0.0993)$ & $(0.0175)$ & $(0.0986)$ \\
\hline$[\mathrm{M} / \mathrm{H}] \log ^{2} \theta$ & 2.3983 & 5.9506 & 4.2027 & 3.4272 & 28.2502 \\
\hline$\sigma$ & $(4.7481)$ & $(2.4610)$ & (2.2530) & $(0.3966)$ & $(2.2373)$ \\
\hline Total $\sigma$ & 149.7603 & 77.6237 & 71.0606 & 12.5094 & 70.5673 \\
\hline $\operatorname{Red} \chi^{2}$ & 0.2127 & 0.0571 & 0.0479 & 0.0015 & 0.0472 \\
\hline Term $(n)$ & Fe5270 & Fe5335 & Fe5406 & Na $D$ & \\
\hline$C_{0}$ & 3.6687 & 4.2195 & 2.2520 & 1.3092 & \\
\hline $\log \theta$ & 26.8280 & 26.0111 & 23.4383 & 21.0015 & \\
\hline$\sigma$ & (1.1715) & $(0.8517)$ & $(0.6402)$ & (1.2074) & \\
\hline $\log ^{2} \theta$ & 44.6660 & 32.4921 & 67.7726 & 152.7714 & \\
\hline$\sigma$ & $(8.6353)$ & $(6.2780)$ & $(4.7191)$ & (8.8997) & \\
\hline $\log ^{3} \theta$ & -205.3421 & -169.5044 & -80.2579 & 412.8384 & \\
\hline$\sigma$ & $(81.3620)$ & (59.1509) & (44.4633) & $(83.8526)$ & \\
\hline$[\mathrm{M} / \mathrm{H}]$ & 1.8747 & 2.2036 & 1.3076 & 1.1254 & \\
\hline$\sigma$ & $(0.1118)$ & $(0.0813)$ & $(0.0611)$ & $(0.1152)$ & \\
\hline$[\mathrm{M} / \mathrm{H}]^{2}$ & 0.3043 & 0.3826 & 0.2548 & 0.2983 & \\
\hline$\sigma$ & $(0.0451)$ & $(0.0328)$ & $(0.0246)$ & $(0.0465)$ & \\
\hline$[\mathrm{M} / \mathrm{H}]^{3}$ & 0.0158 & 0.0222 & 0.0164 & 0.0235 & \\
\hline$\sigma$ & $(0.0050)$ & $(0.0036)$ & $(0.0027)$ & $(0.0051)$ & \\
\hline$[\mathrm{M} / \mathrm{H}] \log \theta$ & 6.5574 & 8.0558 & 8.4043 & 12.2936 & \\
\hline$\sigma$ & $(0.7041)$ & $(0.5119)$ & $(0.3848)$ & $(0.7256)$ & \\
\hline$[\mathrm{M} / \mathrm{H}]^{2} \log \theta$ & 0.3685 & 0.6646 & 0.7870 & 1.4220 & \\
\hline$\sigma$ & (0.1144) & $(0.0831)$ & $(0.0625)$ & (0.1179) & \\
\hline$[\mathrm{M} / \mathrm{H}] \log ^{2} \theta$ & 3.4790 & 2.5911 & 11.0017 & 33.9557 & \\
\hline$\sigma$ & (2.5957) & (1.8871) & (1.4185) & (2.6752) & \\
\hline Total $\sigma$ & 81.8717 & 59.5215 & 44.7419 & 84.3780 & \\
\hline $\operatorname{Red} \chi^{2}$ & 0.0636 & 0.0336 & 0.0190 & 0.0675 & \\
\hline
\end{tabular}

unfortunately, $\mathrm{CaHK}$ is either double-valued as a function of $[\mathrm{M} / \mathrm{H}]$ for $T_{\text {eff }}$ in the range of $4250-5500 \mathrm{~K}$, or becomes negligible and insensitive to $[\mathrm{M} / \mathrm{H}]$ for $[\mathrm{M} / \mathrm{H}] \leqslant-4$ in the $T_{\text {eff }}$ range of $5500-6500 \mathrm{~K}$. We can only recommend $\mathrm{CaHK}$ as a Lick-XMP index for $T_{\text {eff }} \leqslant 4000 \mathrm{~K}$. Because the behavior of CaHK is double valued as a function of both $T_{\text {eff }}$ and $[\mathrm{M} / \mathrm{H}]$ throughout much of the $T_{\mathrm{eff}}-[\mathrm{M} / \mathrm{H}]$ plane, the $C_{\mathrm{n}}$ and partial derivative values are probably not useful for interpolation, and we have not included them.

TiO: We have included the $\mathrm{TiO}_{1}$ and $\mathrm{TiO}_{2}$ indices in our analysis because they are part of the Lick system, but they are only significant in strength and in $[\mathrm{M} / \mathrm{H}]$ sensitivity for the coolest part of our grid, and then only at the highest $[\mathrm{M} / \mathrm{H}]$ values. $\mathrm{TiO}_{2}$ is the stronger of the two indices and remains sensitive to $[\mathrm{M} / \mathrm{H}]$ in the $-2.5-0.0$ range to $T_{\text {eff }}$ values as high as $4250 \mathrm{~K}$. Neither $\mathrm{TiO}$ index has pathologies such as being double-valued. Note that we fit Equation (1) to $\log I$ to account for the strong $T_{\text {eff }}$ and $[\mathrm{M} / \mathrm{H}]$ dependence of $\mathrm{TiO}_{1}$ and $\mathrm{TiO}_{2}$, following W94. Because our grid does not extend to the low $T_{\text {eff }}$ values included in the W94 fit, where TiO is strong, our $C_{\mathrm{n}}$ and partial derivative values are not comparable to W94, and 
Table 5

Same as Table 3, but for Independent Variables $V-K$ and $[\mathrm{M} / \mathrm{H}]$

\begin{tabular}{|c|c|c|c|c|c|}
\hline Polynomial Term & Lick Index, I & & & & \\
\hline Term $(n)$ & $\mathrm{Fe} 4383$ & $\mathrm{Fe} 4531$ & $\mathrm{Fe} 5015$ & $\mathrm{Mg}_{1}$ & $\operatorname{Mg} b$ \\
\hline$C_{0}$ & 2.0247 & 2.0280 & 0.0297 & 0.4264 & 1.9868 \\
\hline$V-K$ & -2.8895 & -0.4509 & 4.5572 & -0.6166 & -3.5266 \\
\hline$\sigma$ & $(1.3722)$ & $(0.8606)$ & $(0.8227)$ & $(0.1854)$ & $(0.4996)$ \\
\hline$V-K^{2}$ & 3.0594 & 1.1931 & -0.3775 & 0.2549 & 1.5847 \\
\hline$\sigma$ & $(0.5385)$ & $(0.3377)$ & $(0.3229)$ & $(0.0728)$ & $(0.1961)$ \\
\hline$V-K^{3}$ & -0.4494 & -0.1639 & 0.0199 & -0.0236 & -0.1178 \\
\hline$\sigma$ & $(0.0657)$ & $(0.0412)$ & $(0.0394)$ & $(0.0089)$ & $(0.0239)$ \\
\hline$[\mathrm{M} / \mathrm{H}]$ & -0.4489 & 0.9816 & 2.2984 & -0.0033 & -0.6624 \\
\hline$\sigma$ & $(0.3320)$ & $(0.2082)$ & $(0.1991)$ & $(0.0449)$ & (0.1209) \\
\hline$[\mathrm{M} / \mathrm{H}]^{2}$ & -0.1629 & 0.2169 & 0.7254 & -0.0077 & -0.1158 \\
\hline$\sigma$ & $(0.0792)$ & $(0.0497)$ & $(0.0475)$ & $(0.0107)$ & $(0.0288)$ \\
\hline$[\mathrm{M} / \mathrm{H}]^{3}$ & -0.0373 & 0.0022 & 0.0540 & -0.0012 & 0.0042 \\
\hline$\sigma$ & $(0.0077)$ & $(0.0048)$ & $(0.0046)$ & $(0.0010)$ & $(0.0028)$ \\
\hline$[\mathrm{M} / \mathrm{H}] V-K$ & 0.9985 & 0.6180 & 1.2306 & -0.0144 & 0.1333 \\
\hline$\sigma$ & $(0.1784)$ & $(0.1119)$ & $(0.1069)$ & $(0.0241)$ & $(0.0649)$ \\
\hline$[\mathrm{M} / \mathrm{H}]^{2} V-K$ & 0.0059 & 0.0097 & 0.0696 & 0.0009 & 0.0749 \\
\hline$\sigma$ & $(0.0158)$ & $(0.0099)$ & $(0.0095)$ & $(0.0021)$ & $(0.0057)$ \\
\hline$[\mathrm{M} / \mathrm{H}] V-K^{2}$ & -0.0728 & -0.0308 & -0.0576 & 0.0109 & 0.1277 \\
\hline$\sigma$ & $(0.0292)$ & $(0.0183)$ & $(0.0175)$ & $(0.0040)$ & $(0.0106)$ \\
\hline Total $\sigma$ & 1.5253 & 0.9566 & 0.9146 & 0.2061 & 0.5554 \\
\hline $\operatorname{Red} \chi^{2}$ & 0.0149 & 0.0059 & 0.0054 & 0.0003 & 0.0020 \\
\hline Term $(n)$ & $\mathrm{Fe} 5270$ & Fe5335 & Fe5406 & $\mathrm{Na} D$ & \\
\hline$C_{0}$ & 2.3521 & 2.1055 & 1.7732 & 2.0387 & \\
\hline$V-K$ & -2.7555 & -2.0371 & -2.7317 & -2.8668 & \\
\hline$\sigma$ & $(0.8051)$ & $(0.5521)$ & $(0.5025)$ & $(0.9161)$ & \\
\hline$V-K^{2}$ & 2.0821 & 1.7716 & 1.7435 & 1.2867 & \\
\hline$\sigma$ & $(0.3159)$ & $(0.2166)$ & $(0.1972)$ & $(0.3595)$ & \\
\hline$V-K^{3}$ & -0.2818 & -0.2483 & -0.2162 & -0.0768 & \\
\hline$\sigma$ & $(0.0386)$ & $(0.0264)$ & $(0.0241)$ & $(0.0439)$ & \\
\hline$[\mathrm{M} / \mathrm{H}]$ & 0.4714 & 0.3801 & -0.0986 & -0.1966 & \\
\hline$\sigma$ & $(0.1948)$ & $(0.1336)$ & $(0.1216)$ & $(0.2217)$ & \\
\hline$[\mathrm{M} / \mathrm{H}]^{2}$ & 0.2452 & 0.2306 & 0.0954 & 0.0009 & \\
\hline$\sigma$ & $(0.0465)$ & $(0.0319)$ & $(0.0290)$ & $(0.0529)$ & \\
\hline$[\mathrm{M} / \mathrm{H}]^{3}$ & 0.0158 & 0.0189 & 0.0119 & 0.0132 & \\
\hline$\sigma$ & $(0.0045)$ & $(0.0031)$ & $(0.0028)$ & $(0.0051)$ & \\
\hline$[\mathrm{M} / \mathrm{H}] V-K$ & 0.6728 & 0.7581 & 0.5120 & 0.1566 & \\
\hline$\sigma$ & $(0.1046)$ & $(0.0718)$ & $(0.0653)$ & $(0.1191)$ & \\
\hline$[\mathrm{M} / \mathrm{H}]^{2} V-K$ & 0.0212 & 0.0406 & 0.0440 & 0.0805 & \\
\hline$\sigma$ & $(0.0092)$ & $(0.0063)$ & $(0.0058)$ & $(0.0105)$ & \\
\hline$[\mathrm{M} / \mathrm{H}] V-K^{2}$ & -0.0390 & -0.0413 & 0.0054 & 0.1307 & \\
\hline$\sigma$ & $(0.0172)$ & $(0.0118)$ & $(0.0107)$ & $(0.0195)$ & \\
\hline Total $\sigma$ & 0.8949 & 0.6137 & 0.5586 & 1.0183 & \\
\hline $\operatorname{Red} \chi^{2}$ & 0.0051 & 0.0024 & 0.0020 & 0.0067 & \\
\hline
\end{tabular}

should be treated with more caution than those of the other indices, and we do not include them here.

\subsection{Partial Derivatives With Respect to $T_{\mathrm{eff}}, V-K$, and $[\mathrm{M} / \mathrm{H}]$}

We have used our $C_{\mathrm{n}}$ values to compute the partial derivatives $\quad 100 \mathrm{~K} \times\left.\frac{\partial I}{\partial T_{\text {eff }}}\right|_{[\mathrm{M} / \mathrm{H}]}, \quad 0.5 \times\left.\frac{\partial I}{\partial[\mathrm{M} / \mathrm{H}]}\right|_{T_{\text {eff }}}$, $0.25 \mathrm{mag} \times\left.\frac{\partial I}{\partial(V-K)}\right|_{[\mathrm{M} / \mathrm{H}]}$, and $0.5 \times\left.\frac{\partial I}{\partial[\mathrm{M} / \mathrm{H}]}\right|_{(V-K)}$ for all Lick indices modeled in LTE, in both index units and in units of $\sigma$, as defined above, for IDS and SDSS resolution spectra. For example, the partial derivative with respect to $\log \theta$ at constant $[\mathrm{M} / \mathrm{H}]$ can be found from

$$
\begin{aligned}
\frac{\partial I}{\partial \log \theta} l_{[\mathrm{M} / \mathrm{H}]} & =C_{2}+2 C_{4} \log \theta+C_{5}\left[\frac{\mathrm{M}}{\mathrm{H}}\right]+ \\
& 3 C_{7} \log ^{2} \theta+C_{8}\left[\frac{\mathrm{M}}{\mathrm{H}}\right]^{2}+2 C_{9} \log \theta\left[\frac{\mathrm{M}}{\mathrm{H}}\right] .
\end{aligned}
$$

Then, the partial derivative with respect to $T_{\text {eff }}$ follows from

$$
\frac{\partial I}{\partial T_{\text {eff }}}=-\frac{\partial I}{\partial \log \theta} \log e / T_{\mathrm{eff}}
$$



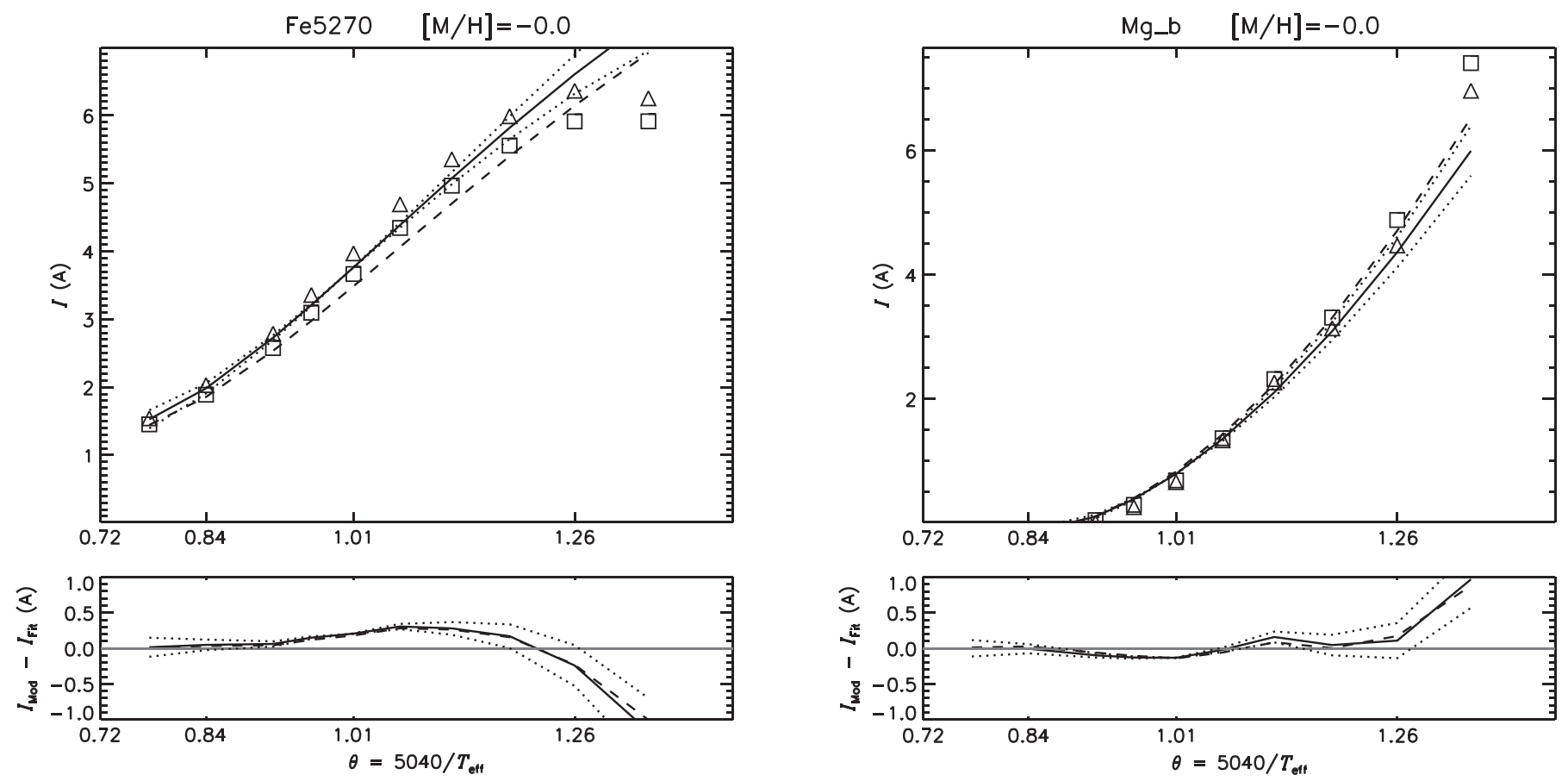

Figure 15. Fe5270: $I(\theta)$ based on multiple linear regression fits for $I(\log \theta)$ at $[\mathrm{M} / \mathrm{H}]=0.0$. Upper panel: fitted relation (solid line) to modeled $I$ values (triangles) at SDSS resolution, and similarly at IDS resolution (dashed line and squares). The dotted lines show the fitted relation for SDSS resolution computed with fitting coefficients, $C_{\mathrm{n}}$, that have had their $1 \sigma$ error from the $\chi^{2}$ fitting procedure (see the text) added and subtracted from them. Lower panel: the residual values for SDSS and IDS resolution (solid and dashed lines, respectively). The dotted lines are the residuals for SDSS resolution for a fitted relation computed with $C_{\mathrm{n}}$ values with their $1 \sigma$ errors added and subtracted (see upper panel caption). The horizontal gray line indicates a residual of zero.
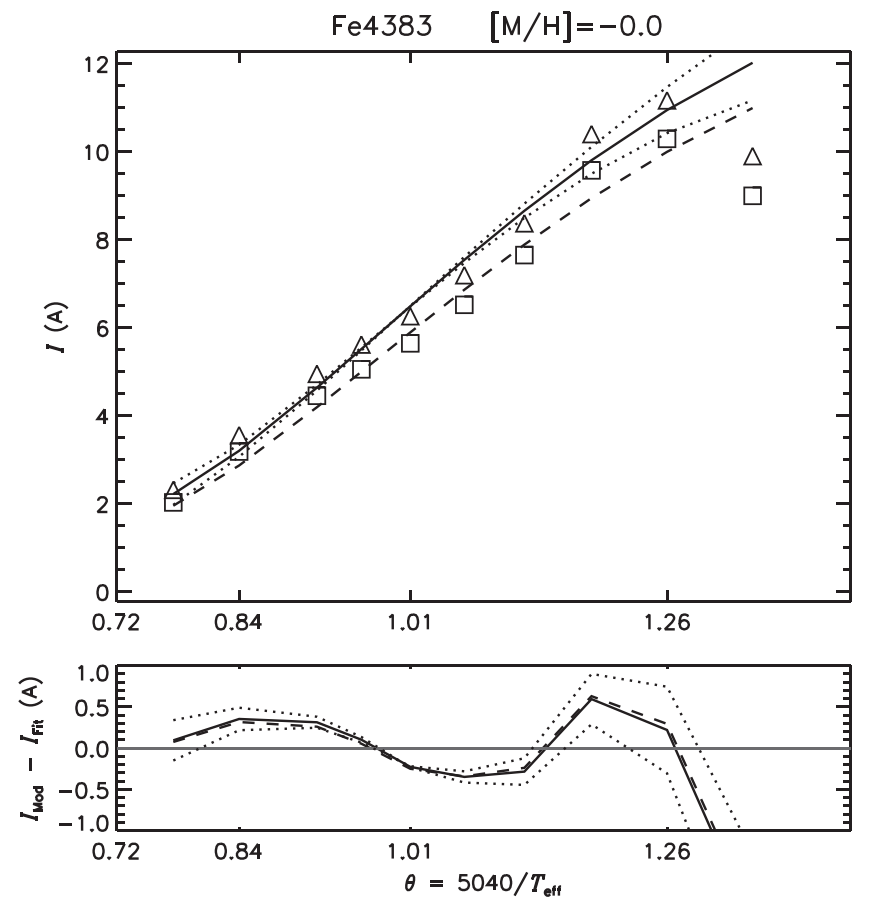

Figure 16. Same as Figure 15, but for Fe4383.

Tables 6 and 7 present the values for our nine identified Lick-XMP indices for SDSS resolution only, and are comparable to Tables 7A and 7B of W94. The values in Table 6,

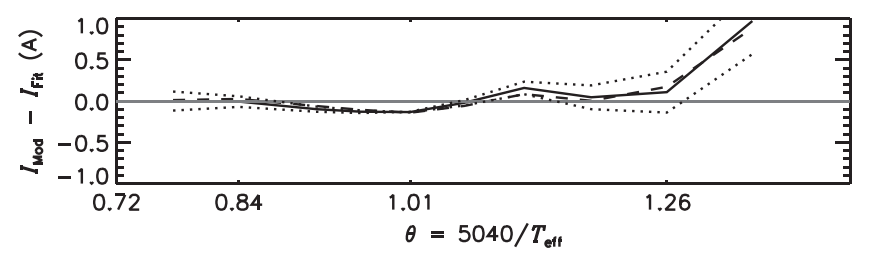

Figure 17. Same as Figure 15, but for $\mathrm{Mg} b$.

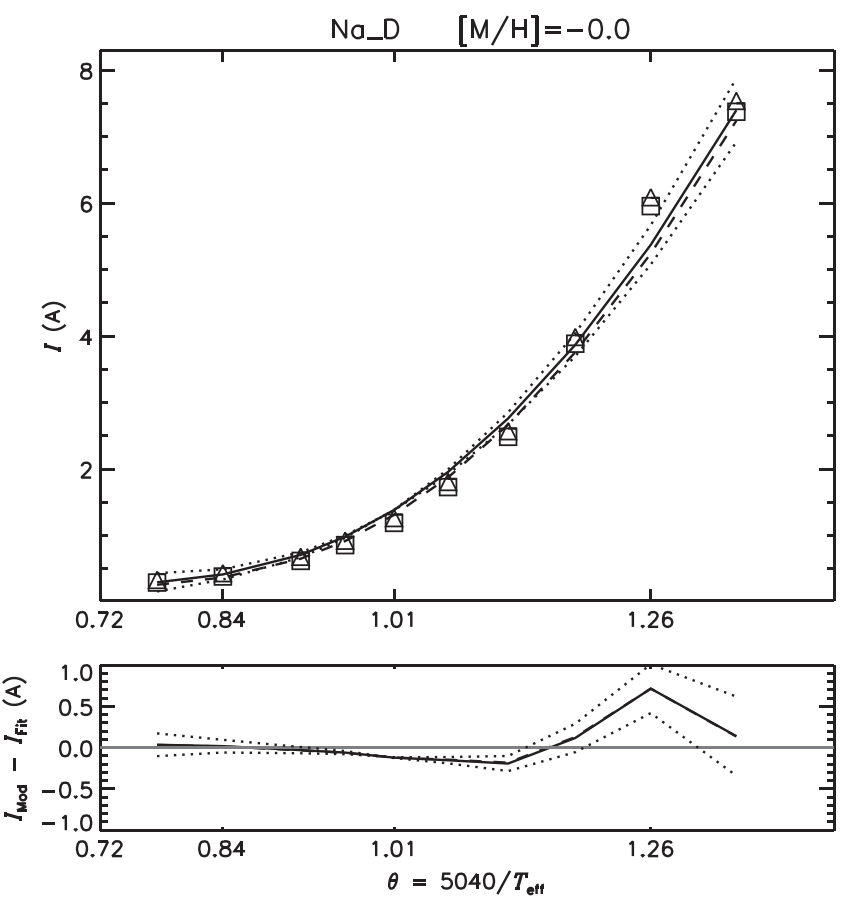

Figure 18. Same as Figure 15, but for Na $D$.

provide an indication of how much the measured value of $I$ will differ between two stars that differ by $\Delta T_{\text {eff }} \approx 100 \mathrm{~K}$ at each value of $[\mathrm{M} / \mathrm{H}]$, and by how much $I$ will differ between two stars that differ by $\Delta[\mathrm{M} / \mathrm{H}] \approx 0.5$ at each value of $T_{\text {eff }}$. Alternately, these derivatives can be used to estimate the change in inferred $T_{\text {eff }}$ or $[\mathrm{M} / \mathrm{H}]$ as a result of the change in computed $I$ caused by NLTE effects (i.e., where $\left.\Delta I \equiv I_{\mathrm{NLTE}}-I_{\mathrm{LTE}}\right)$. The partial derivative values that are in units of $\sigma$ give an indication of the significance, or 

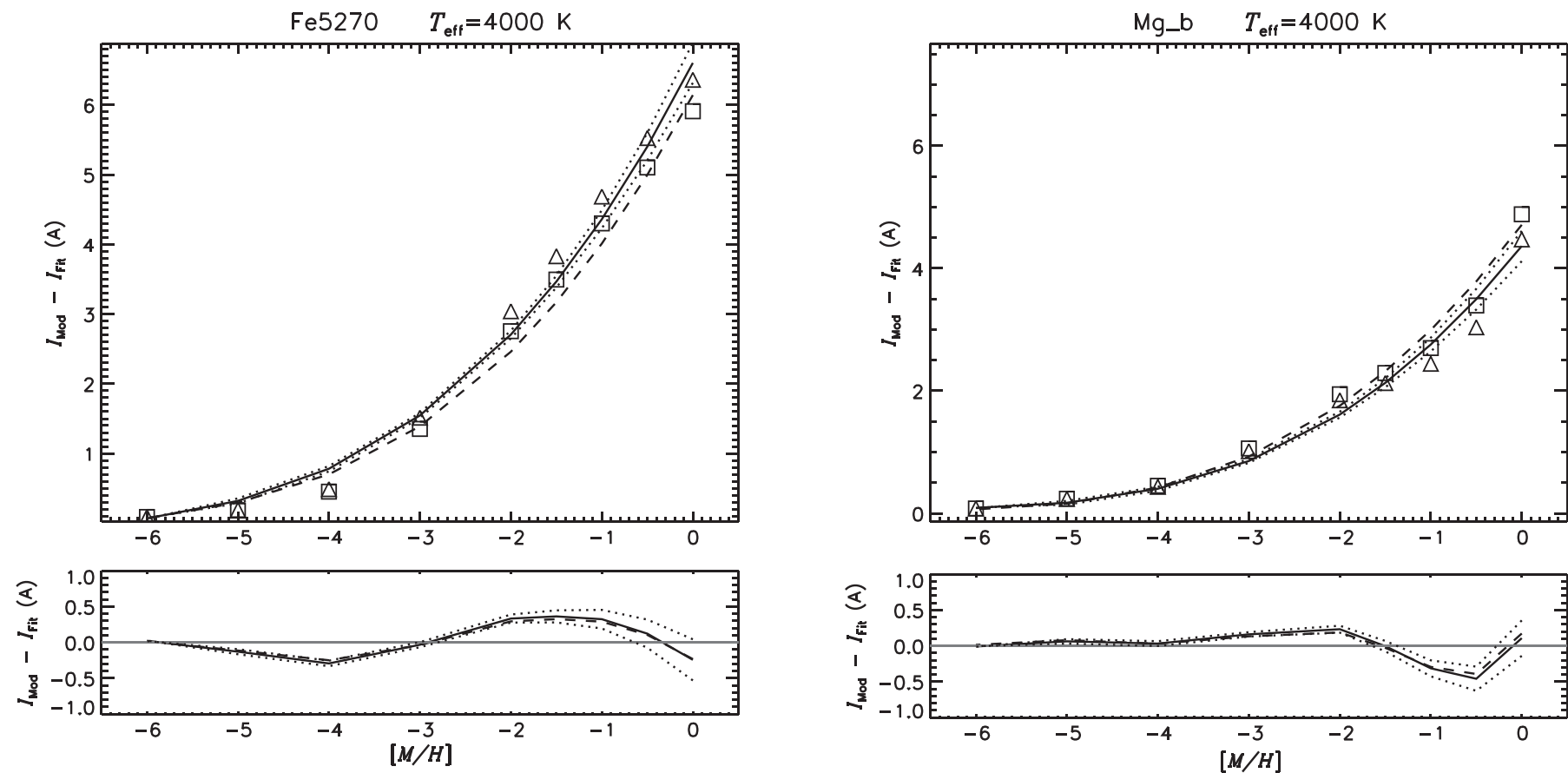

Figure 19. Same as Figure 15, but for the $I([\mathrm{M} / \mathrm{H}])$ relation at $T_{\text {eff }}=4000 \mathrm{~K}$.
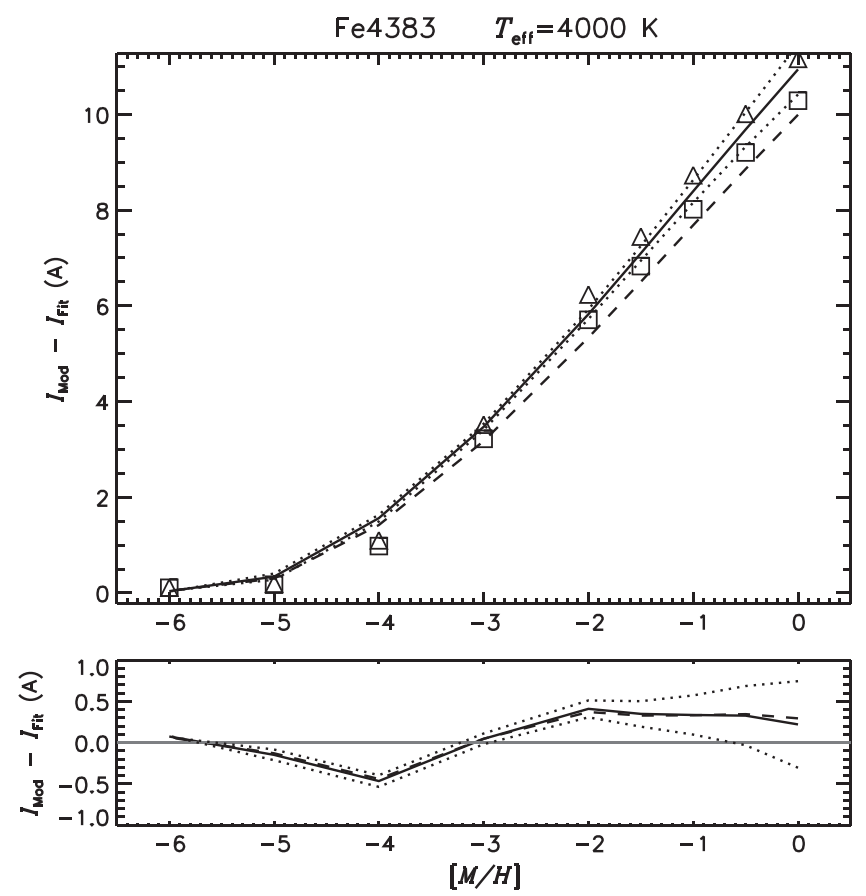

Figure 20. Same as Figure 19, but for Fe4383.

detectability, of these changes in $I$ value given the formal uncertainty $\Delta I \approx \sigma$.

As an example of the implications of NLTE effects for $[\mathrm{M} / \mathrm{H}]$ determination, from Table 6 , for Fe5270 the quantity $0.5 \times\left.\frac{\partial I}{\partial[\mathrm{M} / \mathrm{H}]}\right|_{T_{\text {eff }}}=1.2717$ at $\left(T_{\text {eff }},[\mathrm{M} / \mathrm{H}]\right)=$ $(5000 \mathrm{~K}, 0.0)$, and from Fig. 13, the computed change in $I$ caused by NLTE effects, $\Delta I$, at $5000 \mathrm{~K}$ is $\sim-0.05$. This corresponds to an LTE model $[\mathrm{M} / \mathrm{H}]$ value that is smaller by

$$
\Delta[\mathrm{M} / \mathrm{H}] \approx 0.5 \times-0.05 / 1.2717 \approx-0.02 .
$$

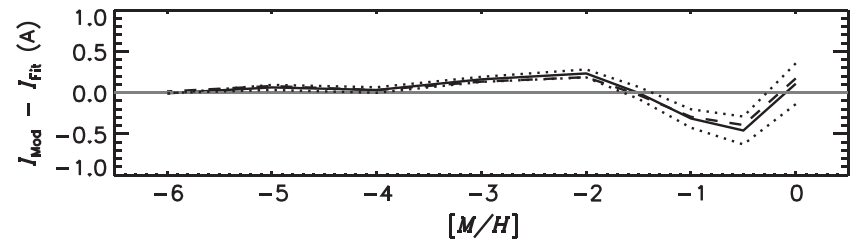

Figure 21. Same as Figure 19, but for $\mathrm{Mg} b$.
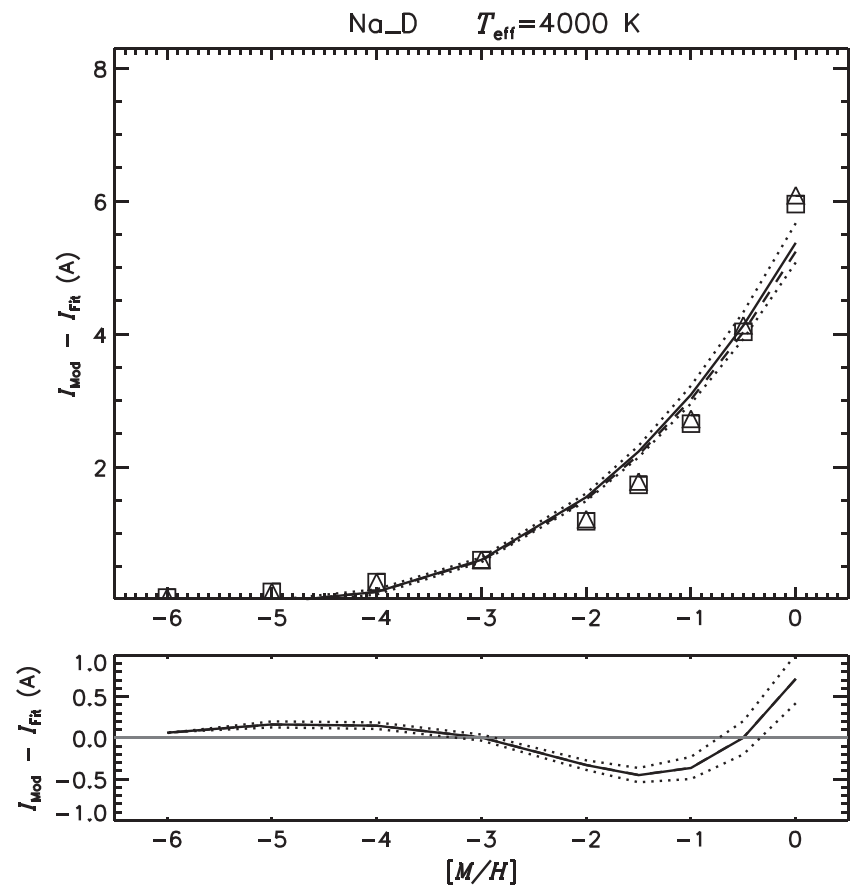

Figure 22. Same as Figure 19, but for Na $D$.

Inversely, fitting a given observed Ivalue with NLTE as compared to LTE models would require a compensating model value of $[\mathrm{M} / \mathrm{H}]$ that is $\sim 0.02$ larger, consistent with the sign of the change in inferred $[\mathrm{M} / \mathrm{H}]$ at fixed $I$ value for $T_{\text {eff }}=4000 \mathrm{~K}$ shown in Figure 5. We emphasize that this is an estimate of $\Delta[\mathrm{M} / \mathrm{H}]$ based on the modeled LTE value of $\frac{\partial I}{\partial[\mathrm{M} / \mathrm{H}]} \mid T_{\text {eff }}$. A more accurate estimate would follow from an NLTE value of $\frac{\partial I}{\partial[\mathrm{M} / \mathrm{H}]} \mid T_{\text {eff }}$, and the importance of this consideration depends 
Table 6

LTE Partial $I$ Derivatives with Respect to $T_{\text {eff }}$ and $[\mathrm{M} / \mathrm{H}]$, at Each Grid Value of $T_{\text {eff }}$ and $[\mathrm{M} / \mathrm{H}]$, in Index Units and in Units of $\sigma$ for SDSS Spectral Resolution for our Nine Lick-XMP Indices

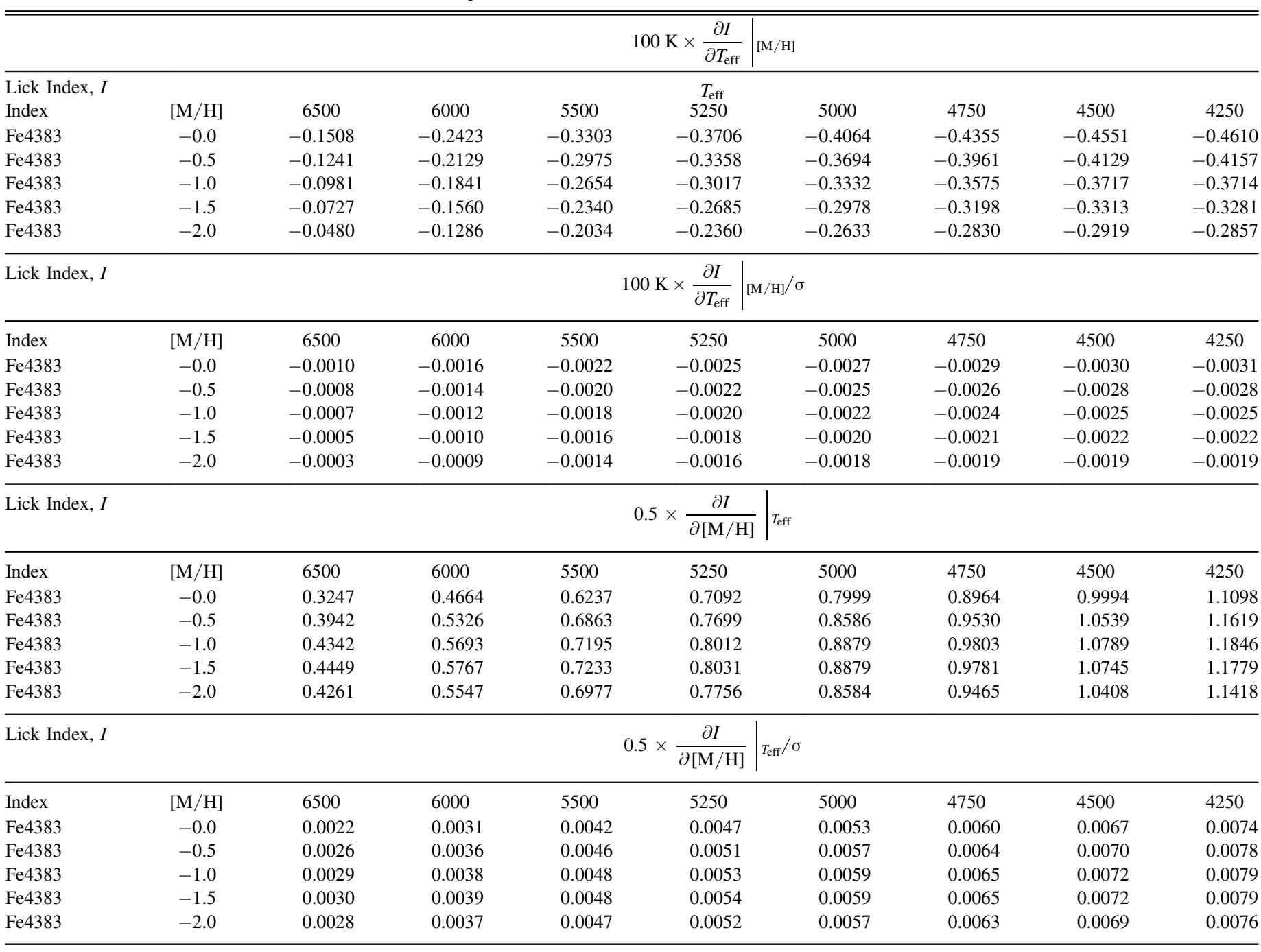

(This table is available in its entirety in machine-readable form.)

on the magnitude of the difference between $\frac{\partial I}{\partial[\mathrm{M} / \mathrm{H}]} \mid T_{\text {eff }}$ values computed from NLTE and LTE model grids. However, computation of NLTE partial derivatives that are comparable to those of LTE requires an NLTE model grid that includes all the same $\left(T_{\text {eff }},[\mathrm{M} / \mathrm{H}]\right)$ points as the LTE grid. Currently, our NLTE model grid covers only a subset because of the larger computational cost of NLTE modeling, and its only purpose is to spot check the effect of NLTE on computed $I$ values at select grid point.

\section{CONCLUSIONS}

Using a grid of red giant synthetic spectra that extends from solar- to XMP-metallicity $([\mathrm{M} / \mathrm{H}]=-6.0)$ we have identified nine of the original 21 Lick indices, designated Lick-XMP indices, that remain significantly detectable and significantly sensitive to $[\mathrm{M} / \mathrm{H}]$ down to XMP values (at least $[\mathrm{M} / \mathrm{H}]=-5.0$ ) for giants of $T_{\mathrm{eff}}<4500 \mathrm{~K}$. For warmer latetype giants, all Lick indices become undetectable or insignificantly sensitive to $[\mathrm{M} / \mathrm{H}]$ before $[\mathrm{M} / \mathrm{H}]$ decreases to -4.0 . The Lick-XMP indices should be the most useful ones for characterizing very old "fossil" stars that formed very early in the history of the Galaxy, and in other galaxies. We also investigated a newer Lick-type index, CaHK, introduced by Serven et al. (2005) and developed by Franchini et al. (2010) as a potential Lick-XMP index, given its strength. However, for $\mathrm{CaHK}, I$ is double valued as a function of $[\mathrm{M} / \mathrm{H}]$ and $T_{\text {eff }}$ over much of our grid and its usefulness is restricted to the cool edge of our grid $\left(T_{\text {eff }}<4000 \mathrm{~K}\right)$.

For our LTE grid of SDSS resolution spectra, we present polynomial coefficients, $C_{\mathrm{n}}$, to third order in the independent variable pairs $(\log \theta,[\mathrm{M} / \mathrm{H}])$ and $((V-K),[\mathrm{M} / \mathrm{H}])$ derived from multi-variate linear regression, approximately comparable to those of W94 for IDS resolution spectra. We present the partial derivatives $\frac{\partial I}{\partial T_{\text {eff }}}\left|[\mathrm{M} / \mathrm{H}], \frac{\partial I}{\partial[\mathrm{M} / \mathrm{H}]}\right|_{T_{\text {eff }}},\left.\frac{\partial I}{\partial(V-K)}\right|_{[\mathrm{M} / \mathrm{H}]}$, and $\frac{\partial I}{\partial[\mathrm{M} / \mathrm{H}]} \mid(V-K)$ computed from our $C_{\mathrm{n}}$ values.

For Fe-dominated Lick indices, the effect of NLTE is to generally weaken the value of $I$ at any given $\left(T_{\text {eff }},[\mathrm{M} / \mathrm{H}]\right)$ value. To put the magnitude of the NLTE effect into context, the change, $\Delta I$, caused by NLTE effects is generally 
Table 7

LTE Partial $I$ Derivatives with Respect to $V-K$ and $[\mathrm{M} / \mathrm{H}]$, at Each Grid Value of $V-K$ for $[\mathrm{M} / \mathrm{H}]=0.0$ Models and [M/H]

\begin{tabular}{|c|c|c|c|c|c|c|c|c|}
\hline & & & & & $\operatorname{ag} \times \frac{\partial I}{-}$ & & & \\
\hline & & & & $0.2 J$ illage & $\overline{\partial(V-K)}$ & {$[\mathrm{M} / \mathrm{H}]$} & & \\
\hline Lick Index, $I$ & & & & $(V-K)$ at $[\mathrm{M} / \mathrm{l}$ & $/ \mathrm{H}]=0.0$ & & & \\
\hline Index & {$[\mathrm{M} / \mathrm{H}]$} & 0.887 & 1.211 & 1.578 & 1.792 & 2.017 & 2.290 & 2.585 \\
\hline $\mathrm{Fe} 4383$ & -0.0 & 0.6126 & 0.8463 & 1.0189 & 1.0744 & 1.0969 & 1.0748 & 0.9900 \\
\hline $\mathrm{Fe} 4383$ & -0.5 & 0.5123 & 0.7459 & 0.9227 & 0.9801 & 1.0083 & 0.9960 & 0.9239 \\
\hline $\mathrm{Fe} 4383$ & -1.0 & 0.4144 & 0.6498 & 0.8299 & 0.8884 & 0.9203 & 0.9136 & 0.8495 \\
\hline $\mathrm{Fe} 4383$ & -1.5 & 0.3137 & 0.5543 & 0.7361 & 0.7977 & 0.8326 & 0.8309 & 0.7770 \\
\hline $\mathrm{Fe} 4383$ & -2.0 & 0.2136 & 0.4555 & 0.6419 & 0.7063 & 0.7449 & 0.7487 & 0.7029 \\
\hline Lick Index, $I$ & & & & $0.25 \mathrm{mag} \times \frac{\partial I}{\partial}$ & & & & \\
\hline & & & & $0.25 \mathrm{mag} \times \overline{\partial(V-}$ & $\overline{K)} \mid[\mathrm{M} / \mathrm{H}] / \sigma$ & & & \\
\hline Index & {$[\mathrm{M} / \mathrm{H}]$} & 0.887 & 1.211 & 1.578 & 1.792 & 2.017 & 2.290 & 2.585 \\
\hline $\mathrm{Fe} 4383$ & -0.0 & -2.8131 & -2.2252 & -1.6230 & -1.3032 & -0.9918 & -0.6481 & -0.3189 \\
\hline $\mathrm{Fe} 4383$ & -0.5 & -2.9024 & -2.3225 & -1.7227 & -1.4147 & -1.1006 & -0.7738 & -0.4398 \\
\hline $\mathrm{Fe} 4383$ & -1.0 & -2.9874 & -2.4089 & -1.8095 & -1.5086 & -1.1932 & -0.8744 & -0.5388 \\
\hline $\mathrm{Fe} 4383$ & -1.5 & -3.0797 & -2.4949 & -1.9022 & -1.5992 & -1.2884 & -0.9726 & -0.6457 \\
\hline $\mathrm{Fe} 4383$ & -2.0 & -3.1715 & -2.5911 & -1.9978 & -1.6955 & -1.3860 & -1.0757 & -0.7484 \\
\hline Lick Index, $I$ & & & & $05 \times \frac{\partial I}{-1}$ & & & & \\
\hline & & & & $0.5 \times \overline{\partial[\mathrm{M} / \mathrm{H}]}$ & $\overline{\mathrm{f}]} \mid(V-K)$ & & & \\
\hline Index & {$[\mathrm{M} / \mathrm{H}]$} & 0.887 & 1.211 & 1.578 & 1.792 & 2.017 & 2.290 & 2.585 \\
\hline $\mathrm{Fe} 4383$ & -0.0 & -1.0540 & -0.8495 & -0.6315 & -0.5151 & -0.3944 & -0.2651 & -0.1258 \\
\hline $\mathrm{Fe} 4383$ & -0.5 & -0.9953 & -0.7911 & -0.5733 & -0.4570 & -0.3365 & -0.2074 & -0.0683 \\
\hline $\mathrm{Fe} 4383$ & -1.0 & -0.9658 & -0.7618 & -0.5442 & -0.4281 & -0.3077 & -0.1787 & -0.0398 \\
\hline $\mathrm{Fe} 4383$ & -1.5 & -0.9654 & -0.7616 & -0.5443 & -0.4283 & -0.3080 & -0.1792 & -0.0405 \\
\hline $\mathrm{Fe} 4383$ & -2.0 & -0.9940 & -0.7905 & -0.5734 & -0.4575 & -0.3374 & -0.2088 & -0.0702 \\
\hline Lick Index, $I$ & & & & $0.5 \times \frac{\partial I}{-}$ & $-(V-K) / \sigma$ & & & \\
\hline & & & &..$\overline{\partial[\mathrm{M} / \mathrm{H}]}$ & $\mid(V-K) / O$ & & & \\
\hline Index & {$[\mathrm{M} / \mathrm{H}]$} & 0.887 & 1.211 & 1.578 & 1.792 & 2.017 & 2.290 & 2.585 \\
\hline $\mathrm{Fe} 4383$ & -0.0 & -0.7299 & -0.5883 & -0.4373 & -0.3567 & -0.2731 & -0.1836 & -0.0871 \\
\hline $\mathrm{Fe} 4383$ & -0.5 & -0.6893 & -0.5479 & -0.3970 & -0.3165 & -0.2330 & -0.1436 & -0.0473 \\
\hline $\mathrm{Fe} 4383$ & -1.0 & -0.6689 & -0.5276 & -0.3769 & -0.2965 & -0.2131 & -0.1238 & -0.0276 \\
\hline $\mathrm{Fe} 4383$ & -1.5 & -0.6686 & -0.5274 & -0.3769 & -0.2966 & -0.2133 & -0.1241 & -0.0280 \\
\hline $\mathrm{Fe} 4383$ & -2.0 & -0.6884 & -0.5474 & -0.3971 & -0.3169 & -0.2337 & -0.1446 & -0.0486 \\
\hline
\end{tabular}

Note. Note that $V-K$ is $[\mathrm{M} / \mathrm{H}]$-dependent and the $V-K$ values used to label the columns are only valid for $[\mathrm{M} / \mathrm{H}]=0.0$ (Figure 4 gives an indication of the $[\mathrm{M} / \mathrm{H}]$ dependence on $V-K$.).

(This table is available in its entirety in machine-readable form.)

comparable to the change that results from computing $I$ from spectra of SDSS resolution rather than that of IDS. The partial derivatives can be used to estimate a change in inferred $T_{\text {eff }}$ at fixed $[\mathrm{M} / \mathrm{H}]$, or a change in inferred $[\mathrm{M} / \mathrm{H}]$ at fixed $T_{\text {eff }}$, resulting from a change in $I$ (e.g., as caused by NLTE effects) at any $\left(T_{\text {eff }},[\mathrm{M} / \mathrm{H}]\right)$ value pair throughout our grid. For example, from Figures 9 and 10, for stars of inferred $T_{\text {eff }} \gtrsim 4200 \mathrm{~K}(\theta \lesssim 1.2)$, an Fe-dominated $I$ value computed in LTE that is too strong might be compensated for by inferring a $T_{\text {eff }}$ value that is too large, for fixed inferred $[\mathrm{M} / \mathrm{H}]$.

C.I.S. is grateful for NSERC Discovery Program grant RGPIN-2014-03979. The calculations were performed with the facilities of the Atlantic Computational Excellence Network (ACEnet).

\section{REFERENCES}

Anderson, L. S. 1989, ApJ, 339, 558

Aoki, W., Beers, T. C., Lee, Y. S., et al. 2013, AJ, 145, 13
Beers, T. C., Preston, G. W., \& Shectman, S. A. 1992, AJ, 103, 1987 Bell, R. A., \& Gustafsson, B. 1989, MNRAS, 236, 653

Belokurov, V. 2013, NewAR, 57, 100

Bergemann, M., Kudritzki, R.-P., Plez, B., et al. 2012, ApJ, 751, 156

Cesetti, M., Pizzella, A., Ivanov, V. D., et al. 2013, A\&A, 549, 129

Cohen, J. G., Christlieb, N., Thompson, D., et al. 2013, ApJ, 778, 56

Franchini, M., Morossi, C., Di Marcantonio, P., Malagnini, M. L., \& Chavez, M. 2010, ApJ, 719, 240

Frebel, A., \& Norris, J. E. 2013, in Planets, Stars and Stellar Systems Vol. 5, ed. T. Oswalt \& G. Gilmore (Berlin: Springer), 55

Gorgas, J., Faber, S. M., Burstein, D., et al. 1993, ApJS, 86, 153

Grevesse, N., \& Sauval, A. J. 1998, SSRv, 85, 161

Hauschildt, P. H., Allard, F., Ferguson, J., Baron, E., \& Alexander, D. R. 1999, ApJ, 525, 871

Ivezic, Z., Beers, T. C., \& Juric, M. 2012, ARA\&A, 50, 251

Johnson, H. L., Mitchell, R. I., Iriarte, B., \& Wisniewski, W. Z. 1966, CoLPL, 4, 99

Le, H. A. N., Kang, W., Pak, S., et al. 2011, arXiv:1108.1499L

Mashonkina, L., Gehren, T., Shi, J.-R., et al. 2011, A\&A, 528, A87

Miller, A. A. 2015, arXiv:1505.01854v1

Mucciarelli, A., Bellazzini, M., Catelan, M., et al. 2013, MNRAS, 435, 3667

Osorio, Y., Barklem, P. S., Lind, K., et al. 2015, arXiv:1504.07593v1

Ridgway, S. T., Joyce, R. R., White, N. M., \& Wing, R. F. 1980, ApJ, 235,126 
Ruchti, G. R., Bergemann, M., Serenelli, A., et al. 2013, MNRAS, 429, 126 Rutten, R. J. 1986, in IAU Coll. 94, Physics of Formation of Fe II Lines Outside LTE, ed. R. Viotti (Dordrecht: Reidel), 185

Schlaufman, K. C., \& Casey, A. R. 2014, ApJ, 797, 13

Serenelli, A. M., Bergemann, M., Ruchti, G., \& Casagrande, L. 2013, MNRAS, 429, 3645

Serven, J., Worthey, G., \& Briley, M. M. 2005, ApJ, 627, 754
Short, C. I., \& Campbell, E. A. 2013, ApJ, 769, 136

Short, C. I., Campbell, E. A., Pickup, H., \& Hauschildt, P. H. 2012, ApJ, 747,143

Short, C. I., \& Hauschildt, P. H. 2009, ApJ, 691, 1634

Worthey, G., Faber, S. M., Jesus Gonzalez, J., \& Burstein, D. 1994, ApJS, 94, 687

Worthey, G., \& Ottaviani, D. L. 1997, ApJS, 111, 377 